\title{
BV minimizers of the area functional in the Heisenberg group under the bounded slope condition
}

\author{
Andrea Pinamonti, Francesco Serra Cassano, Giulia Treu \\ AND DAVIDE VITTONE
}

\begin{abstract}
We consider the area functional for $t$-graphs in the sub-Riemannian Heisenberg group and study minimizers of the associated Dirichlet problem. We prove that, under a bounded slope condition on the boundary datum, there exists a unique minimizer and that this minimizer is Lipschitz continuous. We also provide an example showing that, in the first Heisenberg group, Lipschitz regularity is sharp even under the bounded slope condition.
\end{abstract}

Mathematics Subject Classification (2010): 49Q20 (primary); 53C17, 49Q05 (secondary).

\section{Introduction}

The area functional for the $t$-graph of a function $u \in W^{1,1}(\Omega)$ in the sub-Riemannian Heisenberg group $\mathbb{H}^{n} \equiv \mathbb{R}_{x}^{n} \times \mathbb{R}_{y}^{n} \times \mathbb{R}_{t}$ is

$$
\mathscr{A}(u):=\int_{\Omega}\left|\nabla u+\boldsymbol{X}^{*}\right| \mathrm{d} \mathcal{L}^{2 n},
$$

where $\Omega \subset \mathbb{R}_{x}^{n} \times \mathbb{R}_{y}^{n}$ is an open set and $\boldsymbol{X}^{*}$ is the vector field

$$
\boldsymbol{X}^{*}(x, y):=2(-y, x)
$$

see $[13,46,52]$ for more details. It was shown in [52] that the natural variational setting for this functional is the space $\mathrm{BV}(\Omega)$ of functions with bounded variation in $\Omega$; more precisely, it was proved that the relaxed functional of $\mathscr{A}$ in the $L^{1}$-topology is

$$
\mathscr{A}(u):=\int_{\Omega}\left|\nabla u+X^{*}\right| \mathrm{d} \mathcal{L}^{2 n}+\left|D^{s} u\right|(\Omega), \quad u \in \mathrm{BV}(\Omega),
$$

where $\left|D^{s} u\right|(\Omega)$ is the total variation in $\Omega$ of the singular part of the distributional derivative of $u$.

Received May 14, 2013; accepted in revised version September 26, 2013. 
In this paper, we study the minimizers of $\mathscr{A}$ under Dirichlet boundary conditions

$$
\min \left\{\mathscr{A}(u): u \in \mathrm{BV}(\Omega), u_{\mid \partial \Omega}=\varphi\right\}
$$

where $\Omega$ is assumed to have Lipschitz regular boundary, $u_{\mid \partial \Omega}$ is the trace of $u$ on $\partial \Omega, \varphi \in L^{1}\left(\partial \Omega, \mathcal{H}^{2 n-1}\right)$ is a fixed boundary datum and $\mathcal{H}^{2 n-1}$ denotes the classical Hausdorff measure of dimension $(2 n-1)$. In our main result, Theorem 1.1 below, we prove existence, uniqueness and Lipschitz regularity of minimizers assuming that $\varphi$ satisfies the so-called bounded slope condition (see e.g. [27] or Section 6). We also point out that Lipschitz regularity is sharp at least in the first Heisenberg group $\mathbb{H}^{1}$, see Example 6.6.

Our interest in this problem is twofold. On the one side, it fits in a wellestablished research stream about minimal-surfaces type problems (isoperimetric problem, existence and regularity of $\mathbb{H}$-perimeter minimizing sets, Bernstein problem, etc.) in the Heisenberg group, for which we refer to $[6,7,20-22,34-36,44,45$, 49-52]. On the other side, we attack the problem with typical tools from the Calculus of Variations, using the so called Hilbert-Haar (or "Semi-classical" in [27]) approach. This approach has been recently developed and renewed to study the regularity of minimizers of functionals starting from the regularity of the boundary datum, without assuming either ellipticity or growth conditions on the lagrangian; see $e . g .[2,4,5,8,19,23,38-41]$.

Area minimizers have been widely studied in functional spaces with more regularity than BV. The functional $\mathscr{A}$ has good variational properties such as convexity and lower semicontinuity with respect to the $L^{1}$ topology. On the other hand, it is neither coercive nor differentiable. The lack of differentiability is due to the presence of the so called characteristic points, i.e. the set of points on the graph of $u$ where the tangent plane to the graph coincides with the horizontal plane. Equivalently, the set whose projection on $\mathbb{R}^{2 n}$ is

$$
\operatorname{Char}(u):=\left\{(x, y) \in \Omega: \nabla u(x, y)+\boldsymbol{X}^{*}(x, y)=0\right\} .
$$

Notice that, formally, the Euler equation associated with $\mathscr{A}$ is

$$
\operatorname{div} \frac{\nabla u+X^{*}}{\left|\nabla u+X^{*}\right|}=0 \quad \text { in } \Omega,
$$

which degenerates at points in $\operatorname{Char}(u)$. Clearly, the set Char $(u)$ has a prominent role in studying minimizers' regularity. Several examples of minimizers with at most Lipschitz regularity have been provided in $\mathbb{H}^{1}$, see e.g. $[14,47,48]$; more recently, a non-continuous minimizer was exhibited in [52]. A variety of very interesting results can be found in $[12,13,25,46,51,53]$ (where a priori $C^{2}$ regularity is assumed for minimizers) and in $[15,17]$ (for $C^{1}$ minimizers), also in connection with the Bernstein problem for $t$-graphs. The much more delicate case of minimizers in Sobolev spaces was attacked in [14], where interesting uniqueness and comparison theorems for minimizers in the space $W^{1,2}$ were proved. Uniqueness results for Sobolev minimizers are proved also in [18]. 
Concerning the existence issue, the existence of Lipschitz minimizers for the Dirichlet problem for $\mathscr{A}$ was established in [14], by utilizing an elliptic approximation argument, for $C^{2, \alpha}$-smooth boundary data on $C^{2, \alpha}$-smooth and "parabolicallyconvex" domains. We have also to mention the papers [46] and [16], about which we will say a few words below. Notice that the existence of minimizers is in general not guaranteed even for smooth boundary data on smooth domains, see [52, Example 3.6]. Nevertheless, an existence result (in BV and for any datum $\varphi$ ) was proved in [52] for a penalized functional, see also Section 3.

In this paper, we consider the Dirichlet problem for the functional $\mathscr{A}$ in the space of functions with bounded variation and we study minimizers under the assumption that the boundary datum $\varphi$ satisfies a bounded slope condition with constant $Q$ ( $Q$-B.S.C. for short). This approach is inspired by some classical and wellknown results in the Calculus of Variations that go back to Hilbert and Haar ([33] and [28] respectively) and has been used by Hartman, Nirenberg and Stampacchia [29-32,54]. The main classical result in this framework (see e.g. [27, Chapter 1] or, for minimal surfaces, [43]) states that, given a strictly convex integral functional depending only on the gradient and a boundary datum satisfying the $Q$ B.S.C., there exists a unique minimizer in the class of Lipschitz functions and its Lipschitz constant is not greater than $Q$. We stress that, in the general statement in [27], neither growth assumptions nor ellipticity conditions are required whereas these hypotheses usually play a crucial role for existence and regularity results. In the same setting, it has been recently proved in [8] that any continuous minimizer in the Sobolev space $W^{1,1}$ is Lipschitz continuous with Lipschitz constant not greater than $Q$.

The main tools used to prove these results are: the validity of comparison principles between minimizers, the invariance of minimizers under translations of the domain, and a Haar-Radò type theorem stating that the maximum among the difference quotients of the minimizer is attained at the boundary. Subsequent papers $[39,40]$ addressed the problem of considering functionals that are not strictly convex. The main difficulty, in this case, is that comparison principles do not hold in their generality (an example can be found in [9] or in [42]) and it is overcome by detecting special minimizers which instead satisfy them, see [9-11,42]. It is worth remarking that, in all these papers, there are assumptions guaranteeing the boundedness of the faces of the epigraph of the lagrangian. Concerning functionals depending also on lower order terms, this approach works for lagrangians of sum type as $f(\xi)+g(z, u)$, see $[3,4,10,23]$.

In the present paper we use some of the techniques described above but we encounter new difficulties that we briefly sketch here and will be discussed in details in the following sections. First of all, we deal with functions of bounded variation and we use ideas of [24], where functionals depending only on the gradient are considered. The second point is the dependance of our functional on points of $\Omega$ encoded in the vector field $\boldsymbol{X}^{*}$. Moreover, the epigraph of our lagrangian has unbounded faces. All these peculiarities led us to face many new technical problems that will be considered in Sections 4 and 5. 
In the framework of the first Heisenberg group $\mathbb{H}^{1}$, the bounded slope condition was already considered in [46] by S. D. Pauls. Using an approximation scheme by means of minimal surfaces in the Riemannian approximations of $\mathbb{H}^{n}$, he showed the existence in $W^{1, p}(\Omega) \cap C^{0}(\Omega)$ of weak solutions to the Euler equation associated with $\mathscr{A}$.

Before stating our main result let us underline some peculiarities about the bounded slope condition. On the one hand, it is a quite restrictive assumption because it implies that, unless $\varphi$ is affine, $\Omega$ is convex. On the other hand, the class of functions satisfying it is quite large, since M. Miranda [43] proved that, if $\Omega$ is uniformly convex, then any $\varphi \in \mathcal{C}^{1,1}$ satisfies the $Q$-B.S.C. for some $Q$.

Theorem 1.1. Let $\Omega \subset \mathbb{R}^{2 n}$ be open, bounded and with Lipschitz regular boundary, and let $\varphi: \partial \Omega \rightarrow \mathbb{R}$ satisfy the $Q-B . S . C$.for some $Q>0$. Then, the minimization problem

$$
\min \left\{\mathscr{A}(u): u \in \mathrm{BV}(\Omega), u_{\mid \partial \Omega}=\varphi\right\}
$$

admits a unique solution $\hat{u}$. Moreover, $\hat{u}$ is Lipschitz continuous and $\operatorname{Lip}(\hat{u}) \leq$ $Q+4 \sup _{z \in \Omega}|z|$.

Notice that, if $\varphi$ satisfies the B.S.C., then it is Lipschitz continuous on $\partial \Omega$ : in this sense, our assumptions on the boundary datum are stronger than those in $[16$, Theorem A], where the authors prove the existence and the continuity of BV minimizers on $C^{2, \alpha}$ parabolically convex domains assuming only the continuity of the boundary datum. Nevertheless, we are able to obtain stronger results (namely: uniqueness and Lipschitz regularity of the minimizer) on (possibly) less regular domains. In fact our result applies, in particular, when $\Omega$ is uniformly convex and $\varphi$ is (the restriction to $\partial \Omega$ of) a function of class $\mathcal{C}^{1,1}$ : in this case, as previously mentioned, $\varphi$ automatically satisfies the B.S.C.

We conjecture that, as [16, Theorem A], our main result holds as well for more general functionals.

We also want to stress a couple of interesting points concerning Theorem 1.1. First, in contrast with the Semi-Classical Theory, the Lipschitz constant of the minimizer may a priori be greater than the constant $Q$ given by the bounded slope condition. Second, Theorem 1.1 is sharp at least in $\mathbb{H}^{1}$ in the sense that, even under the bounded slope condition, a minimizer might not be better than Lipschitz continuous, see Example 6.6.

The proof of Theorem 1.1 is based on several intermediate results which have an independent interest. We mention, for instance, a Comparison Principle for minimizers, Theorem 4.5, which in turn is based on the existence of the (pointwise a.e.) "maximum" and "minimum" in the family of minimizers, see Proposition 4.4. The uniqueness in Theorem 1.1 is based on a criterion stated in Proposition 5.1 (for which we have to credit [14]) and on the fact that affine functions are the unique minimizers under their own boundary datum, see Theorem 5.5.

The structure of the paper is the following. Section 2 contains basic facts about functions with bounded variation and their traces. In Section 3 we recall several preliminary results about the functional $\mathscr{A}$. Section 4 is devoted to the study of the 
set of minimizers and its structure, with particular regard to comparison principles. In Section 5 we prove the uniqueness results in Proposition 5.1 and Theorem 5.5. Finally, Section 6 is devoted to the proof of Theorem 1.1 .

\section{Functions of bounded variation and traces}

The aim of this section is to recall some basic properties of the space of functions of bounded variation; we refer to the monographs $[1,26]$ for a more extensive account on the subject as well as for proofs of the results we are going to recall here. $\Omega$ if

Let $\Omega$ be an open set in $\mathbb{R}^{n}$. We say that $u \in L^{1}(\Omega)$ has bounded variation in

$$
\sup \left\{\int_{\Omega} u \operatorname{div} \varphi \mathrm{d} \mathcal{L}^{n}: \varphi \in C_{c}^{1}(\Omega),\|\varphi\| \leq 1\right\}<+\infty
$$

equivalently, if there exist a $\mathbb{R}^{n}$-valued Radon measure $D u:=\left(D_{1} u, \ldots, D_{n} u\right)$ in $\Omega$ which represents the distributional derivative of $u$, i.e., if

$$
\int_{\Omega} u \frac{\partial \varphi}{\partial x_{i}} \mathrm{~d} \mathcal{L}^{n}=-\int_{\Omega} \varphi \mathrm{d} D_{i} u \quad \forall \varphi \in C_{c}^{1}(\Omega), \forall i=1, \ldots, n .
$$

The space of functions with bounded variation in $\Omega$ is denoted by $\mathrm{BV}(\Omega)$. By definition, $W^{1,1}(\Omega) \subset \operatorname{BV}(\Omega)$ and $D u=\nabla u \mathcal{L}^{n}$ for any $u \in W^{1,1}(\Omega)$.

We denote by $|D u|$ the total variation of the measure $D u ;|D u|$ defines a finite measure on $\Omega$ and the supremum in (2.1) coincides with $|D u|(\Omega)$. It is well-known that $\mathrm{BV}(\Omega)$ is a Banach space when endowed with the norm

$$
\|u\|_{\mathrm{BV}}:=\|u\|_{L^{1}}+|D u|(\Omega) .
$$

By the Radon-Nikodym Theorem, if $u \in \mathrm{BV}(\Omega)$ one can write $D u=D^{a} u+D^{s} u$, where $D^{a} u$ is the absolutely continuous part of $D u$ with respect to $\mathcal{L}^{n}$ and $D^{s} u$ is the singular part of $D u$ with respect to $\mathcal{L}^{n}$. We denote by $\nabla u \in L^{1}(\Omega)$ the density of $D^{a} u$ with respect to $\mathcal{L}^{n}$, so that $D^{a} u=\nabla u \mathcal{L}^{n}$. It turns out that, if $u \in \operatorname{BV}(\Omega)$, then $u$ is approximately differentiable at a.e. $x \in \Omega$ with approximate differential $\nabla u(x)$, i.e.,

$$
\lim _{\rho \rightarrow 0^{+}} f_{B(x, \rho)} \frac{|u(y)-\tilde{u}(x)-\langle\nabla u(x), y-x\rangle|}{\rho} \mathrm{d} \mathcal{L}^{n}=0 \quad \text { for } \mathcal{L}^{n} \text {-a.e. } x \in \Omega \text {. }
$$

We now recall a few basic facts about boundary trace properties for BV functions. Assume that $\Omega \subset \mathbb{R}^{n}$ is a bounded open set with Lipschitz regular boundary; the spaces $L^{p}(\partial \Omega), p \in[1,+\infty]$, will be always understood with respect to the (finite) measure $\mathcal{H}^{n-1}\left\llcorner\partial \Omega\right.$, where $\mathcal{H}^{n-1}$ denotes the $(n-1)$-dimensional Hausdorff measure on $\mathbb{R}^{n}$ (see again [1] or [26]). It is well-known that for any $u \in \mathrm{BV}(\Omega)$ there exists a (unique) function $u_{\mid \partial \Omega} \in L^{1}(\partial \Omega)$ such that, for $\mathcal{H}^{n-1}$-a.e. $x \in \partial \Omega$,

$$
\lim _{\rho \rightarrow 0^{+}} \rho^{-n} \int_{\Omega \cap B(x, \rho)}\left|u-u_{\mid \partial \Omega}(x)\right| \mathrm{d} \mathcal{L}^{n}=\lim _{\rho \rightarrow 0^{+}} f_{\Omega \cap B(x, \rho)}\left|u-u_{\mid \partial \Omega}(x)\right| \mathrm{d} \mathcal{L}^{n}=0 .
$$


The function $u_{\mid \partial \Omega}$ is called the trace of $u$ on $\partial \Omega$. The trace operator $u \mapsto u_{\mid \partial \Omega}$ is linear and continuous between $\left(\mathrm{BV}(\Omega),\|\cdot\|_{\mathrm{BV}}\right)$ and $L^{1}(\partial \Omega)$; actually, it is continuous also when $\mathrm{BV}(\Omega)$ is endowed with the (weaker) topology induced by the so-called strict convergence, see [1, Definition 3.14].

Remark 2.1. It is well-known that, if $u_{1}, u_{2} \in \mathrm{BV}(\Omega)$, then $\bar{u}:=\max \left\{u_{1}, u_{2}\right\}$ and $\underline{u}:=\min \left\{u_{1}, u_{2}\right\}$ belong to $\mathrm{BV}(\Omega)$; moreover, one can show that

$$
\bar{u}_{\mid \partial \Omega}=\max \left\{u_{1 \mid \partial \Omega}, u_{2 \mid \partial \Omega}\right\}, \quad \underline{u}_{\mid \partial \Omega}=\min \left\{u_{1 \mid \partial \Omega}, u_{2 \mid \partial \Omega}\right\} .
$$

The proof of this fact follows in a standard way from the very definition of traces.

Since $D u \ll|D u|$ we can write $D u=\sigma_{u}|D u|$ for a $|D u|$-measurable function $\sigma_{u}: \Omega \rightarrow \mathbb{S}^{n-1}$. With this notation, one has also

$$
\begin{array}{r}
\int_{\Omega} u \operatorname{div} \varphi \mathrm{d} \mathcal{L}^{n}=-\int_{\Omega}\left\langle\sigma_{u}, \varphi\right\rangle \mathrm{d}|D u|+\int_{\partial \Omega} u \mid \partial \Omega\left\langle\varphi, v_{\Omega}\right\rangle \mathrm{d} \mathcal{H}^{n-1} \\
\forall \varphi \in C_{c}^{1}\left(\mathbb{R}^{n}, \mathbb{R}^{n}\right)
\end{array}
$$

where $\nu_{\Omega}$ is the unit outer normal to $\partial \Omega$.

Finally, we recall the following fact, whose proof stems from (2.2).

Proposition 2.2 ([26, Remark 2.13]). Assume that $\Omega$ and $\Omega_{0}$ are open subsets of $\mathbb{R}^{n}$ with bounded Lipschitz boundary and such that $\Omega \Subset \Omega_{0}$. If $u \in \mathrm{BV}(\Omega)$ and $v \in \mathrm{BV}\left(\Omega_{0} \backslash \bar{\Omega}\right)$, then the function

$$
f(x):= \begin{cases}u(x) & \text { if } x \in \Omega \\ v(x) & \text { if } x \in \Omega_{0} \backslash \bar{\Omega}\end{cases}
$$

belongs to $\mathrm{BV}\left(\Omega_{0}\right)$ and

$$
|D f|(\partial \Omega)=\int_{\partial \Omega}\left|u_{\mid \partial \Omega}-v_{\mid \partial \Omega}\right| \mathrm{d} \mathcal{H}^{n-1},
$$

where we have used the notation $v_{\mid \partial \Omega}$ to mean $\left(v_{\mid \partial\left(\Omega_{0} \backslash \bar{\Omega}\right)}\right)\llcorner\partial \Omega$.

\section{The area functional for $t$-graphs in the Heisenberg group}

Before introducing the area functional $\mathscr{A}$ with more details, we need some preliminary notation. For $z:=(x, y) \in \mathbb{R}^{n} \times \mathbb{R}^{n}$ we define

$$
z^{*}:=(-y, x) \in \mathbb{R}^{2 n}
$$

Let us state some useful properties of the map $z \mapsto z^{*}$; we denote by $\cdot, \nabla$ and div the standard scalar product, gradient and divergence in $\mathbb{R}^{2 n}$. 
Lemma 3.1. The following properties hold:

(i) if $z_{1}, z_{2} \in \mathbb{R}^{2 n}$ are linearly dependent, then $z_{1} \cdot z_{2}^{*}=0$;

(ii) $z_{1} \cdot z_{2}=z_{1}^{*} \cdot z_{2}^{*}$ for each $z_{1}, z_{2} \in \mathbb{R}^{2 n}$;

(iii) if $\Omega \subset \mathbb{R}^{2 n}$ is open and $f \in C^{\infty}(\Omega)$, then $\operatorname{div}(\nabla f)^{*}=0$ on $\Omega$.

Proof. The first two statements are straightforward. To prove (iii), observe that $(\nabla f)^{*}=\left(-\partial_{n+1} f, \ldots,-\partial_{2 n} f, \partial_{1} f, \ldots, \partial_{n} f\right)$, thus

$$
\operatorname{div}(\nabla f)^{*}=-\sum_{i=1}^{n} \partial_{i} \partial_{n+i} f+\sum_{i=1}^{n} \partial_{n+i} \partial_{i} f=0 .
$$

Given an open set $\Omega \subset \mathbb{R}^{2 n}$ we define the convex functional $\mathscr{A}_{\Omega}: \mathrm{BV}(\Omega) \rightarrow \mathbb{R}$

$$
\mathscr{A}_{\Omega}(u):=\int_{\Omega}\left|\nabla u+\boldsymbol{X}^{*}\right| \mathrm{d} \mathcal{L}^{2 n}+\left|D^{s} u\right|(\Omega),
$$

where $\boldsymbol{X}(z):=2 z$ and, clearly, $\boldsymbol{X}^{*}(z)=2 z^{*}$.

When the open set $\Omega$ is clear from the context, we will simply write $\mathscr{A}$ instead of $\mathscr{A}_{\Omega}$. Using the standard identification of the Heisenberg group $\mathbb{H}^{n}$ with $\mathbb{R}_{z}^{2 n} \times \mathbb{R}_{t}$, there holds

$$
\mathscr{A}(u)=\left|\partial E_{u}^{t}\right|_{\mathbb{H}}(\Omega \times \mathbb{R}),
$$

where $\left|\partial E_{u}^{t}\right| \mathbb{H}(\Omega \times \mathbb{R})$ denotes the $\mathbb{H}$-perimeter in $\Omega \times \mathbb{R} \subset \mathbb{H}^{n}$ of the $t$-subgraph

$$
E_{u}^{t}:=\left\{(z, t) \in \mathbb{H}^{n}: z \in \Omega, t<u(z)\right\}
$$

of $u$. See [52] for more details. It was proved in [52] that $\mathscr{A}$ is lower semicontinuous with respect to the $L^{1}$-convergence and

$$
\mathscr{A}(u)=\inf \left\{\liminf _{j \rightarrow \infty} \int_{\Omega}\left|\nabla u_{j}+X^{*}\right| \mathrm{d} \mathcal{L}^{2 n}:\left(u_{j}\right)_{j \in \mathbb{N}} \subset C^{1}(\Omega), u_{j} \rightarrow u \text { in } L^{1}(\Omega)\right\} .
$$

The following approximation result holds:

Proposition 3.2. Let $\Omega \subset \mathbb{R}^{2 n}$ be a bounded open set with Lipschitz boundary. Let $u \in \mathrm{BV}(\Omega)$ with $u_{\mid \partial \Omega}=\varphi \in L^{1}(\Omega)$; then there exists a sequence $\left(u_{k}\right)_{k} \subset C^{\infty}(\Omega)$ converging to $u$ in $L^{1}(\Omega)$ and such that

$$
\begin{aligned}
& \left(u_{k}\right)_{\mid \partial \Omega}=\varphi \quad \forall k \in \mathbb{N}, \\
& \mathscr{A}_{\Omega}(u)=\lim _{k \rightarrow \infty} \int_{\Omega}\left|\nabla u_{k}+X^{*}\right| \mathrm{d} \mathcal{L}^{2 n} .
\end{aligned}
$$


Proof. The existence of a sequence $\left(u_{k}\right)_{k} \subset C^{\infty}(\Omega)$ converging to $u$ in $L^{1}(\Omega)$ and such that (3.3) holds was proved in [52, Theorem 3.2], see also [52, Corollary 3.3]. More precisely, this sequence was constructed in Step 4 of the proof of [52, Theorem 3.2] by imposing certain conditions on suitably mollified functions, see formulae (3.5)-(3.7) therein. Reasoning as in [26, Remark 1.18], it can be proved that condition (3.5) of [52] implies that

$$
\lim _{\rho \rightarrow 0^{+}} \rho^{-n} \int_{\Omega \cap B(z, \rho)}\left|u-u_{k}\right| \mathrm{d} \mathcal{L}^{2 n}=0 \quad \text { for } \mathcal{H}^{2 n-1} \text {-a.e. } z \in \partial \Omega
$$

and (3.2) follows from the definition of traces.

We are interested in the existence of minimizers for $\mathscr{A}$ under prescribed boundary conditions. Assuming $\Omega$ to be a bounded domain with Lipschitz boundary, we consider

$$
M_{1}:=\inf \left\{\mathscr{A}(u): u \in \mathrm{BV}(\Omega), u_{\mid \partial \Omega}=\varphi\right\} .
$$

It is known that the infimum $M_{1}$ might not be attained even for smooth $\Omega$ and $\varphi$, see [52, Example 3.6].

On the other hand, one can consider the functional

$$
\mathscr{A}_{\varphi, \Omega}(u):=\mathscr{A}_{\Omega}(u)+\int_{\partial \Omega}\left|u_{\mid \partial \Omega}-\varphi\right| \mathrm{d} \mathcal{H}^{2 n-1}
$$

where the integral on the right hand side can be seen as a penalization for $u$ not taking the boundary value $\varphi$; this penalization is natural from the viewpoint of the geometry of $\mathbb{H}^{n}$ as shown in [52, Remark 3.8]. Again, we will simply write $\mathscr{A}_{\varphi}$ instead of $\mathscr{A}_{\varphi, \Omega}$ when the open set $\Omega$ is clear from the context. By using the Direct Method of the Calculus of Variations (see again [52]), it can be shown that the problem

$$
M_{2}:=\min \left\{\mathscr{A}_{\varphi}(u): u \in \mathrm{BV}(\Omega)\right\}
$$

admits always a solution.

Let us show that the Lavrentiev phenomenon does not occur for our minimization problem.

Proposition 3.3. Let $\Omega \subset \mathbb{R}^{2 n}$ be a bounded open set with Lipschitz regular boundary and $\varphi$ be in $L^{1}(\partial \Omega)$; then, setting

$$
M_{3}:=\inf \left\{\mathscr{A}(u): u \in C^{\infty}(\Omega) \cap W^{1,1}(\Omega), u_{\mid \partial \Omega}=\varphi\right\}
$$

we have $M_{1}=M_{2}=M_{3}$, where $M_{1}$ and $M_{2}$ are defined above.

Proof. Clearly, one has $M_{3} \geq M_{1} \geq M_{2}$ because

$$
\left\{u \in C^{\infty}(\Omega) \cap W^{1,1}(\Omega): u_{\mid \partial \Omega}=\varphi\right\} \subset\left\{u \in \mathrm{BV}(\Omega): u_{\mid \partial \Omega}=\varphi\right\} \subset \mathrm{BV}(\Omega)
$$


and $\mathscr{A}_{\varphi}$ coincides with $\mathscr{A}$ on $\left\{u \in \mathrm{BV}(\Omega): u_{\mid \partial \Omega}=\varphi\right\}$. Let $u \in \mathrm{BV}(\Omega)$ with $u_{\mid \partial \Omega}=\varphi$ and consider $\left(u_{k}\right)_{k} \subset C^{\infty}(\Omega)$ as in Proposition 3.2. Then

$$
M_{3} \leq \mathscr{A}(u)=\lim _{k \rightarrow \infty} \mathscr{A}\left(u_{k}\right),
$$

which implies $M_{3} \leq M_{1}$ and hence $M_{3}=M_{1}$. Finally, the equality $M_{1}=M_{2}$ has been established in [52, Theorem 1.4], and the proof is accomplished.

Remark 3.4. One can easily show that, if the boundary datum $\varphi$ is Lipschitz continuous on $\partial \Omega$, then the equalities

$$
M_{1}=M_{2}=M_{3}=\inf \left\{\mathscr{A}(u): u \in \operatorname{Lip}(\Omega), u_{\mid \partial \Omega}=\varphi\right\}
$$

hold.

\section{The set of minimizers and Comparison Principles}

The aim of this section is to establish a Comparison Principle for minimizers of the area functional with penalization on the boundary. It is well-known that Comparison Principles are strictly related to uniqueness of solutions and that functionals defined in the BV space do not exhibit, in general, uniqueness of minimizers, even in the case of a strictly convex lagrangian. In our case, the lagrangian $f(z, \xi):=\left|\xi+X^{*}(z)\right|$ is not even strictly convex. The validity of Comparison Principles for non strictly convex functionals has been studied in $[9,11,37,40,42]$ in the case of superlinear growth, and it has been proved for special classes of minimizers. In this section we follow the same ideas, but we have to overcome some new difficulties that are related both to the properties of $\mathrm{BV}$ and to the fact that the lagrangian depends also on the variable $z$.

The main Comparison Principle is stated in Theorem 4.5. Its proof is based on several steps and, in particular, it relies on some inequalities that, in our opinion, have an interest on their own. For this reason, we enunciate them as separate propositions. The proof of Theorem 4.5 will then follow in a few lines.

We remark that similar results for functionals with linear growth, depending just on the gradient and defined in the space of function of bounded variation, have been recently obtained in [24].

The next two propositions state two inequalities between the values of the area functional at $u_{1}, u_{2}, u_{1} \vee u_{2}:=\sup \left\{u_{1}, u_{2}\right\}$ and $u_{1} \wedge u_{2}:=\inf \left\{u_{1}, u_{2}\right\}$. The first one is stated for the area functional and the second one is for the functional with the penalization on the boundary. We observe that, when one deals with integral functionals defined in Sobolev spaces, these inequalities turn out to be equalities, whose proof is straightforward (see [42, Lemma 5.1]).

Proposition 4.1. Let $\Omega \subset \mathbb{R}^{2 n}$ be a bounded open set with Lipschitz, boundary. Let $u_{1}, u_{2} \in \mathrm{BV}(\Omega)$ be fixed. Then

$$
\mathscr{A}\left(u_{1} \vee u_{2}\right)+\mathscr{A}\left(u_{1} \wedge u_{2}\right) \leq \mathscr{A}\left(u_{1}\right)+\mathscr{A}\left(u_{2}\right) .
$$


Proof. Recalling (3.1), inequality (4.1) is equivalent to

$\left|\partial E_{u_{1} \vee u_{2}}^{t}\right|_{\mathbb{H}}(\Omega \times \mathbb{R})+\left|\partial E_{u_{1} \wedge u_{2}}^{t}\right|_{\mathbb{H}}(\Omega \times \mathbb{R}) \leq\left|\partial E_{u_{1}}^{t}\right|_{\mathbb{H}}(\Omega \times \mathbb{R})+\left|\partial E_{u_{2}}^{t}\right|_{\mathbb{H}}(\Omega \times \mathbb{R})$

which follows from [52, Proposition 2.3] on noticing that

$$
E_{u_{1} \vee u_{2}}^{t}=E_{u_{1}}^{t} \cup E_{u_{2}}^{t}, \quad E_{u_{1} \wedge u_{2}}^{t}=E_{u_{1}}^{t} \cap E_{u_{2}}^{t} .
$$

The next proposition is the analogue of the previous one for the functional $\mathscr{A}_{\varphi}$ where the boundary conditions are taken into account.

Proposition 4.2. Let $\Omega \subset \mathbb{R}^{2 n}$ be a bounded open set with Lipschitz regular boundary. Then, for each $u_{1}, u_{2} \in \mathrm{BV}(\Omega)$ and $\varphi_{1}, \varphi_{2} \in L^{1}(\partial \Omega)$ we have

$$
\mathscr{A}_{\varphi_{1} \vee \varphi_{2}, \Omega}\left(u_{1} \vee u_{2}\right)+\mathscr{A}_{\varphi_{1} \wedge \varphi_{2}, \Omega}\left(u_{1} \wedge u_{2}\right) \leq \mathscr{A}_{\varphi_{1}, \Omega}\left(u_{1}\right)+\mathscr{A}_{\varphi_{2}, \Omega}\left(u_{2}\right)
$$

Proof. Let us fix a bounded open set $\Omega_{0} \subset \mathbb{R}^{2 n}$ with Lipschitz boundary and such that $\Omega \Subset \Omega_{0}$. By [26, Theorem 2.16] it is possible to find $f_{1}, f_{2}$ in $W^{1,1}\left(\Omega_{0} \backslash \bar{\Omega}\right)$ such that

$$
f_{1 \mid \partial \Omega}=\varphi_{1} \quad \text { and } \quad f_{2 \mid \partial \Omega}=\varphi_{2} .
$$

Define

$$
v_{1}:=\left\{\begin{array}{ll}
u_{1} & \text { in } \Omega \\
f_{1} & \text { in } \Omega_{0} \backslash \bar{\Omega},
\end{array} \quad v_{2}:= \begin{cases}u_{2} & \text { in } \Omega \\
f_{2} & \text { in } \Omega_{0} \backslash \bar{\Omega}\end{cases}\right.
$$

so that

$$
v_{1} \vee v_{2}=\left\{\begin{array}{ll}
u_{1} \vee u_{2} & \text { in } \Omega \\
f_{1} \vee f_{2} & \text { in } \Omega_{0} \backslash \bar{\Omega},
\end{array} \quad v_{1} \wedge v_{2}= \begin{cases}u_{1} \wedge u_{2} & \text { in } \Omega \\
f_{1} \wedge f_{2} & \text { in } \Omega_{0} \backslash \bar{\Omega} .\end{cases}\right.
$$

We have $v_{1}, v_{2}, v_{1} \vee v_{2}, v_{1} \wedge v_{2} \in \mathrm{BV}\left(\Omega_{0}\right)$ and Lemma 4.1 gives

$$
\mathscr{A}_{\Omega_{0}}\left(v_{1} \vee v_{2}\right)+\mathscr{A}_{\Omega_{0}}\left(v_{1} \wedge v_{2}\right) \leq \mathscr{A}_{\Omega_{0}}\left(v_{1}\right)+\mathscr{A}_{\Omega_{0}}\left(v_{2}\right) \text {. }
$$

Writing $\left(f_{1} \vee f_{2}\right)_{\mid \partial \Omega}$ for $\left(\left(f_{1} \vee f_{2}\right)_{\mid \partial\left(\Omega_{0} \backslash \bar{\Omega}\right)}\right)\llcorner\partial \Omega$, we have by Proposition 2.2 and Remark 2.1

$$
\begin{aligned}
& \mathscr{A}_{\Omega_{0}}\left(v_{1} \vee v_{2}\right) \\
&=\int_{\Omega_{0}}\left|\nabla\left(v_{1} \vee v_{2}\right)+\boldsymbol{X}^{*}\right| \mathrm{d} \mathcal{L}^{2 n}+\left|D^{s}\left(v_{1} \vee v_{2}\right)\right|\left(\Omega_{0}\right) \\
&=\mathscr{A}_{\Omega}\left(v_{1} \vee v_{2}\right)+\mathscr{A}_{\Omega_{0} \backslash \bar{\Omega}}\left(v_{1} \vee v_{2}\right)+\left|D^{s}\left(v_{1} \vee v_{2}\right)\right|(\partial \Omega) \\
&=\mathscr{A}_{\Omega}\left(u_{1} \vee u_{2}\right)+\mathscr{A}_{\Omega_{0} \backslash \bar{\Omega}}\left(f_{1} \vee f_{2}\right) \\
&+\int_{\partial \Omega}\left|\left(u_{1} \vee u_{2}\right)_{\mid \partial \Omega}-\left(f_{1} \vee f_{2}\right)\right| \partial \Omega \mid \mathrm{d} \mathcal{H}^{2 n-1} \\
&= \mathscr{A}_{\Omega}\left(u_{1} \vee u_{2}\right)+\int_{\Omega_{0} \backslash \bar{\Omega}}\left|\nabla\left(f_{1} \vee f_{2}\right)+\boldsymbol{X}^{*}\right| \mathrm{d} \mathcal{L}^{2 n} \\
&+\int_{\partial \Omega}\left|\left(u_{1} \vee u_{2}\right)_{\mid \partial \Omega}-\left(\varphi_{1} \vee \varphi_{2}\right)\right| \mathrm{d} \mathcal{H}^{2 n-1} \\
&= \mathscr{A}_{\varphi_{1} \vee \varphi_{2}, \Omega}\left(u_{1} \vee u_{2}\right)+\int_{\Omega_{0} \backslash \bar{\Omega}}\left|\nabla\left(f_{1} \vee f_{2}\right)+\boldsymbol{X}^{*}\right| \mathrm{d} \mathcal{L}^{2 n}
\end{aligned}
$$


where we also utilized Remark 2.1. In a similar way one obtains

$$
\begin{aligned}
& \mathscr{A}_{\Omega_{0}}\left(v_{1} \wedge v_{2}\right)=\mathscr{A}_{\varphi_{1} \wedge \varphi_{2}, \Omega}\left(\left(u_{1} \wedge u_{2}\right)\right)+\int_{\Omega_{0} \backslash \bar{\Omega}}\left|\nabla\left(f_{1} \wedge f_{2}\right)+X^{*}\right| \mathrm{d} \mathcal{L}^{2 n} \\
& \mathscr{A}_{\Omega_{0}}\left(v_{1}\right)=\mathscr{A}_{\varphi_{1}, \Omega}\left(u_{1}\right)+\int_{\Omega_{0} \backslash \bar{\Omega}}\left|\nabla f_{1}+\boldsymbol{X}^{*}\right| \mathrm{d} \mathcal{L}^{2 n} \\
& \mathscr{A}_{\Omega_{0}}\left(v_{2}\right)=\mathscr{A}_{\varphi_{2}, \Omega}\left(u_{2}\right)+\int_{\Omega_{0} \backslash \bar{\Omega}}\left|\nabla f_{2}+\boldsymbol{X}^{*}\right| \mathrm{d} \mathcal{L}^{2 n} .
\end{aligned}
$$

Now, (4.2) will follow from (4.3), (4.4) and (4.5) provided we show that

$$
\begin{aligned}
& \int_{\Omega_{0} \backslash \bar{\Omega}}\left|\nabla\left(f_{1} \vee f_{2}\right)+\boldsymbol{X}^{*}\right| \mathrm{d} \mathcal{L}^{2 n}+\int_{\Omega_{0} \backslash \bar{\Omega}}\left|\nabla\left(f_{1} \wedge f_{2}\right)+\boldsymbol{X}^{*}\right| \mathrm{d} \mathcal{L}^{2 n} \\
= & \int_{\Omega_{0} \backslash \bar{\Omega}}\left|\nabla f_{1}+\boldsymbol{X}^{*}\right| \mathrm{d} \mathcal{L}^{2 n}+\int_{\Omega_{0} \backslash \bar{\Omega}}\left|\nabla f_{2}+\boldsymbol{X}^{*}\right| \mathrm{d} \mathcal{L}^{2 n} .
\end{aligned}
$$

This can be easily seen by using the well-known facts

$$
\begin{aligned}
& \nabla\left(f_{1} \vee f_{2}\right)=\left(\nabla f_{1}\right) \chi_{\left\{f_{1} \geq f_{2}\right\}}+\left(\nabla f_{2}\right) \chi_{\left\{f_{1}<f_{2}\right\}}, \\
& \nabla\left(f_{1} \wedge f_{2}\right)=\left(\nabla f_{2}\right) \chi_{\left\{f_{1} \geq f_{2}\right\}}+\left(\nabla f_{1}\right) \chi_{\left\{f_{1}<f_{2}\right\}} .
\end{aligned}
$$

The proof is accomplished.

Given a bounded open set $\Omega \subset \mathbb{R}^{2 n}$ with Lipschitz regular boundary and a function $\varphi \in L^{1}(\partial \Omega)$ we define

$$
\mathscr{M}_{\varphi}:=\underset{u}{\arg \min } \mathscr{A}_{\varphi, \Omega}(u)=\underset{u}{\arg \min }\left\{\mathscr{A}_{\Omega}(u)+\int_{\partial \Omega}|u-\varphi| \mathrm{d} \mathcal{H}^{2 n-1}\right\} .
$$

The set $\mathscr{M}_{\varphi} \subset \mathrm{BV}(\Omega)$ is not empty by [52, Theorem 1.4$]$.

Corollary 4.3. Let $\varphi_{1}, \varphi_{2} \in L^{1}(\partial \Omega)$ be such that $\varphi_{1} \leq \varphi_{2} \mathcal{H}^{2 n-1}$-a.e. on $\partial \Omega$ and assume that $u_{1} \in \mathscr{M}_{\varphi_{1}}$ and $u_{2} \in \mathscr{M}_{\varphi_{2}}$. Then $\left(u_{1} \vee u_{2}\right) \in \mathscr{M}_{\varphi_{2}}$ and $\left(u_{1} \wedge u_{2}\right) \in$ $\mathscr{M}_{\varphi_{1}}$.

Proof. The assumptions that $u_{2}$ is a minimizer of $\mathscr{A}_{\varphi_{2}, \Omega}$ and $\varphi_{1} \leq \varphi_{2}$ imply that

$$
\mathscr{A}_{\varphi_{2}, \Omega}\left(u_{1} \vee u_{2}\right) \geq \mathscr{A}_{\varphi_{2}, \Omega}\left(u_{2}\right) .
$$

Analogously we have

$$
\mathscr{A}_{\varphi_{1}, \Omega}\left(u_{1} \wedge u_{2}\right) \geq \mathscr{A}_{\varphi_{1}, \Omega}\left(u_{1}\right) .
$$

By Proposition 4.2 it follows that

$$
\mathscr{A}_{\varphi_{1}, \Omega}\left(u_{1} \vee u_{2}\right)+\mathscr{A}_{\varphi_{2}, \Omega}\left(u_{1} \wedge u_{2}\right)=\mathscr{A}_{\varphi_{1}, \Omega}\left(u_{1}\right)+\mathscr{A}_{\varphi_{2}, \Omega}\left(u_{2}\right)
$$

so that equality holds both in (4.6) and in (4.7). 
In [40] it has been proved that the set of minimizers of a superlinear convex functional has a maximum $\bar{u}$ (respectively a minimum $u$ ) defined as the pointwise supremum (infimum) of the minimizers. These special minimizers are then used to prove one-sided Comparison Principles. Now, with a different technique required by the use of functions of bounded variation, we prove a similar result.

Proposition 4.4. Let $\Omega \subset \mathbb{R}^{2 n}$ be a bounded open set with Lipschitz regular boundary and let $\varphi \in L^{1}(\partial \Omega)$. Then, there exists $\bar{u}, \underline{u} \in \mathscr{M}_{\varphi}$ such that the inequalities

$$
\underline{u} \leq u \leq \bar{u} \quad \mathcal{L}^{2 n} \text {-a.e. in } \Omega
$$

hold for any $u \in \mathscr{M}_{\varphi}$.

Proof. We start by proving that $\mathscr{M}_{\varphi}$ is bounded in $\mathrm{BV}(\Omega)$. Given $u \in \mathscr{M}_{\varphi}$, we define $J:=\mathscr{A}_{\varphi, \Omega}(u)<\infty$; clearly, $J$ depends only on $\varphi$ and not on $u$. We have

$$
\begin{aligned}
|D u|(\Omega)= & \int_{\Omega}|\nabla u| \mathrm{d} \mathcal{L}^{2 n}+\left|D^{s} u\right|(\Omega) \\
\leq & \int_{\Omega}\left|\nabla u+\boldsymbol{X}^{*}\right| \mathrm{d} \mathcal{L}^{2 n} \\
& \quad+\int_{\Omega}\left|\boldsymbol{X}^{*}\right| \mathrm{d} \mathcal{L}^{2 n}+\left|D^{s} u\right|(\Omega)+\int_{\partial \Omega}|u-\varphi| \mathrm{d} \mathcal{H}^{2 n-1} \\
= & +\int_{\Omega}\left|\boldsymbol{X}^{*}\right| \mathrm{d} \mathcal{L}^{2 n}<\infty .
\end{aligned}
$$

Moreover, by [26, Theorem 1.28 and Remark 2.14] there exists $c=c(n)>0$ such that

$$
\begin{aligned}
\|u\|_{L^{1}(\Omega)} & \leq|\Omega|^{1 / 2 n}\|u\|_{L^{2 n /(2 n-1)}(\Omega)} \\
& \leq c|\Omega|^{1 / 2 n}\left(|D u|(\Omega)+\int_{\partial \Omega}|u| \partial \Omega \mid \mathrm{d} \mathcal{H}^{2 n-1}\right) \\
& \leq c|\Omega|^{1 / 2 n}\left(|D u|(\Omega)+\int_{\partial \Omega}|u| \partial \Omega-\varphi\left|\mathrm{d} \mathcal{H}^{2 n-1}+\int_{\partial \Omega}\right| \varphi \mid \mathrm{d} \mathcal{H}^{2 n-1}\right) \\
& =c|\Omega|^{1 / 2 n}\left(J+\int_{\partial \Omega}|\varphi| \mathrm{d} \mathcal{H}^{2 n-1}\right)
\end{aligned}
$$

where $|\Omega|:=\mathcal{L}^{2 n}(\Omega)$. This, together with (4.9), implies that $\mathscr{M}_{\varphi}$ is bounded in $\mathrm{BV}(\Omega)$.

Therefore (see [1, Theorem 3.23]), $\mathscr{M}_{\varphi}$ is pre-compact in $L^{1}(\Omega)$, i.e., for every sequence $\left(u_{h}\right)_{h \in \mathbb{N}} \subset \mathscr{M}_{\varphi}$ there exist $u \in \mathrm{BV}(\Omega)$ and a subsequence $\left(u_{h_{k}}\right)_{k \in \mathbb{N}}$ such that $u_{h_{k}} \rightarrow u$ in $L^{1}(\Omega)$. Since $\mathscr{A}_{\varphi, \Omega}$ is lower semicontinuous with respect to the $L^{1}$-convergence we have also

$$
\mathscr{A}_{\varphi, \Omega}(u) \leq \liminf _{k \rightarrow \infty} \mathscr{A}_{\varphi, \Omega}\left(u_{h_{k}}\right)=J
$$


We deduce that $u \in \mathscr{M}_{\varphi}$, i.e., that $\mathscr{M}_{\varphi}$ is indeed compact in $L^{1}(\Omega)$. Now, the functional

$$
\mathrm{BV}(\Omega) \ni u \longmapsto I(u):=\int_{\Omega} u \mathrm{~d} \mathcal{L}^{2 n}
$$

is continuous in $L^{1}(\Omega)$, hence it admits maximum $\bar{u}$ and minimum $\underline{u}$ in $\mathscr{M}_{\varphi}$ : let us prove that $\bar{u}, \underline{u}$ satisfy (4.8) for any $u \in \mathscr{M}_{\varphi}$.

Assume by contradiction there exists $u \in \mathscr{M}_{\varphi}$ such that $\Omega^{\prime}:=\{z \in \Omega$ : $u(z)>\bar{u}(z)\}$ has strictly positive measure. Then, by Corollary $4.3, u \vee \bar{u}$ is in $\mathscr{M}_{\varphi}$. Moreover

$$
\int_{\Omega}(u \vee \bar{u}) \mathrm{d} \mathcal{L}^{2 n}=\int_{\Omega^{\prime}} u \mathrm{~d} \mathcal{L}^{2 n}+\int_{\Omega \backslash \Omega^{\prime}} \bar{u} \mathrm{~d} \mathcal{L}^{2 n}>\int_{\Omega} \bar{u} \mathrm{~d} \mathcal{L}^{2 n}
$$

yielding a contradiction. The fact that $u \geq \underline{u}$ follows in a similar way.

Now we can state a Comparison Principle inspired by the results obtained in [40] for superlinear functionals in Sobolev spaces.

Theorem 4.5. Let $\Omega \subset \mathbb{R}^{2 n}$ be a bounded open set with Lipschitz regular boundary; let $\varphi, \psi \in L^{1}(\partial \Omega)$ be such that $\varphi \leq \psi \mathcal{H}^{2 n-1}$-a.e. on $\partial \Omega$. Consider the functions $\bar{u}, \underline{u} \in \mathscr{M}_{\varphi}$ and $\bar{w}, \underline{w} \in \mathscr{M}_{\psi}$ such that ${ }^{1}$

$$
\begin{array}{ll}
\underline{u} \leq u \leq \bar{u} & \mathcal{L}^{2 n} \text {-a.e. in } \Omega, \forall u \in \mathscr{M}_{\varphi} \\
\underline{w} \leq w \leq \bar{w} & \mathcal{L}^{2 n} \text {-a.e. in } \Omega, \forall w \in \mathscr{M}_{\psi} .
\end{array}
$$

Then

$$
\bar{u} \leq \bar{w} \quad \text { and } \quad \underline{u} \leq \underline{w} \quad \mathcal{L}^{2 n} \text {-a.e. in } \Omega
$$

and, in particular,

$$
\begin{array}{ll}
u \leq \bar{w} & \mathcal{L}^{2 n} \text {-a.e. in } \Omega, \forall u \in \mathscr{M}_{\varphi} \\
\underline{u} \leq w & \mathcal{L}^{2 n} \text {-a.e. in } \Omega, \forall w \in \mathscr{M}_{\psi} .
\end{array}
$$

Proof. We have proved in Corollary 4.3 that $\bar{w} \vee \bar{u}$ is a minimizer of $\mathscr{A}_{\psi}$ and $\bar{w} \wedge \bar{u}$ is a minimizer of $\mathscr{A}_{\varphi}$. Assumption (4.10) then gives (4.11), which allows us to conclude.

The next result is a consequence of the Comparison Principle. We state it here explicitly since, in this formulation, it will be useful in the sequel.

Corollary 4.6. Let $\Omega \subset \mathbb{R}^{2 n}$ be a bounded open set with Lipschitz regular boundary and $\varphi, \psi \in L^{\infty}(\partial \Omega)$; let $\bar{u}, \underline{u} \in \mathscr{M}_{\varphi}$ and $\bar{w}, \underline{w} \in \mathscr{M}_{\psi}$ be as in (4.10). Then, for every $\alpha \in \mathbb{R}$, one has

$$
\begin{aligned}
& \bar{u}+\alpha, \underline{u}+\alpha \in \mathscr{M}_{\varphi+\alpha} \\
& \underline{u}+\alpha \leq u \leq \bar{u}+\alpha \quad \mathcal{L}^{2 n} \text {-a.e. in } \Omega, \forall u \in \mathscr{M}_{\varphi+\alpha}
\end{aligned}
$$

${ }^{1}$ The existence of $\bar{u}, \underline{u}, \bar{w}, \underline{w}$ is guaranteed by Proposition 4.4. 
and

$$
\begin{aligned}
\|\bar{u}-\bar{w}\|_{L^{\infty}(\Omega)} & \leq\|\varphi-\psi\|_{L^{\infty}(\partial \Omega)} \\
\|\underline{u}-\underline{w}\|_{L^{\infty}(\Omega)} & \leq\|\varphi-\psi\|_{L^{\infty}(\partial \Omega)} .
\end{aligned}
$$

In particular, the implications

$$
\begin{aligned}
& \bar{u}_{\mid \partial \Omega}=\varphi, \bar{w}_{\mid \partial \Omega}=\psi \quad \Rightarrow \quad\|\bar{u}-\bar{w}\|_{L^{\infty}(\Omega)}=\|\varphi-\psi\|_{L^{\infty}(\partial \Omega)} \\
& \underline{u}_{\mid \partial \Omega}=\varphi, \underline{w}_{\mid \partial \Omega}=\psi \quad \Rightarrow \quad\|\underline{u}-\underline{w}\|_{L^{\infty}(\Omega)}=\|\varphi-\psi\|_{L^{\infty}(\partial \Omega)},
\end{aligned}
$$

hold.

Proof. The statements in (4.12) follow at once on noticing that

$$
\mathscr{A}_{\varphi+\alpha, \Omega}(u+\alpha)=\mathscr{A}_{\varphi, \Omega}(u) \quad \forall u \in \mathrm{BV}(\Omega) .
$$

Let $\alpha:=\|\varphi-\psi\|_{L^{\infty}(\partial \Omega)} \in \mathbb{R}$, then

$$
\varphi \leq \psi+\alpha \quad \mathcal{H}^{2 n-1} \text {-a.e. in } \partial \Omega,
$$

and, by (4.12) and Corollary 4.5, we get

$$
\bar{u} \leq \bar{w}+\alpha \text { and } \underline{u} \leq \underline{w}+\alpha \quad \mathcal{L}^{2 n} \text {-a.e. in } \Omega .
$$

An analogous argument shows that

$$
\bar{w} \leq \bar{u}+\alpha \text { and } \underline{w} \leq \underline{u}+\alpha \quad \mathcal{L}^{2 n} \text {-a.e. in } \Omega,
$$

whence (4.13).

If the assumptions in (4.14) are satisfied, classical properties of traces ensure that the reverse inequalities in (4.13) holds, and this gives the validity of the implications in (4.14).

\section{Uniqueness of special minimizers}

This section is devoted to some uniqueness results for minimizers of the area functional. We have already recalled that, in general, minimizers of functionals defined in BV are not unique. Comparison principles are particularly interesting in this context. If we consider the functional with the penalization on the boundary, whenever we detect a special boundary datum yielding uniqueness of the minimizer we also know that this minimizer satisfies the Comparison Principle, so that it can be used as a 'barrier'. We emphasize this fact in Corollary 5.6, that will be the key point in the proof of the main result of this paper.

The following uniqueness result can be proved on combining Theorems 5.1, 5.2 and 5.3 in [14]. For the reader's benefit, we give here a slightly simplified proof. 
Proposition 5.1. Let $\Omega \subset \mathbb{R}^{2 n}$ be a bounded open set with Lipschitz regular boundary; fix $p \in[1,2]$ and set $p^{\prime}:=\frac{p}{p-1} \in[2,+\infty]$. Let $\varphi \in W^{1, p^{\prime}}(\Omega)$ be fixed and consider the minimization problem

$$
\min \left\{\mathscr{A}(u): u \in \varphi+W_{0}^{1, p}(\Omega)\right\} .
$$

If $u \in W^{1, p^{\prime}}(\Omega)$ and $v \in W^{1, p}(\Omega)$ are minimizers of (5.1), then

$$
u=v \quad \mathcal{L}^{2 n} \text {-a.e. in } \Omega .
$$

Proof. Let us consider the function $(u+v) / 2 \in \varphi+W_{0}^{1, p}(\Omega)$; we claim that

$$
\mathscr{A}\left(\frac{u+v}{2}\right)=\frac{1}{2}(\mathscr{A}(u)+\mathscr{A}(v)) .
$$

Indeed, the convexity of $\mathscr{A}$ gives

$$
\mathscr{A}\left(\frac{u+v}{2}\right) \leq \frac{1}{2}(\mathscr{A}(u)+\mathscr{A}(v)),
$$

while the reverse inequality follows from the fact that $u$ and $v$ are minimizers for the problem (5.1). This proves (5.2), whence

$$
\int_{\Omega}\left|\frac{1}{2} \nabla u+\frac{1}{2} \nabla v+\boldsymbol{X}^{*}\right| \mathrm{d} \mathcal{L}^{2 n}=\frac{1}{2} \int_{\Omega}\left|\nabla u+\boldsymbol{X}^{*}\right| \mathrm{d} \mathcal{L}^{2 n}+\frac{1}{2} \int_{\Omega}\left|\nabla v+\boldsymbol{X}^{*}\right| \mathrm{d} \mathcal{L}^{2 n} .
$$

This in turn implies that

$$
\left|\left(\nabla u+X^{*}\right)+\left(\nabla v+X^{*}\right)\right|=\left|\nabla u+X^{*}\right|+\left|\nabla v+X^{*}\right| \quad \text { a.e. in } \Omega,
$$

i.e., $\nabla u+\boldsymbol{X}^{*}$ and $\nabla v+\boldsymbol{X}^{*}$ are parallel (and with the same direction) $\mathcal{L}^{2 n}$-a.e. in $\Omega$. In particular, by Lemma 3.1 (i) we obtain

$$
0=\left(\nabla u+X^{*}\right)^{*} \cdot\left(\nabla v+X^{*}\right)=\left((\nabla u)^{*}-X\right) \cdot\left(\nabla v+X^{*}\right) \quad \text { a.e. in } \Omega .
$$

Thus

$$
\int_{\Omega_{1}}\left((\nabla u)^{*}-\boldsymbol{X}\right) \cdot\left(\nabla v+\boldsymbol{X}^{*}\right) \mathrm{d} \mathcal{L}^{2 n}=0,
$$

where $\Omega_{1}:=\{z \in \Omega: u(z)>v(z)\}$. Let us expand (5.3) to get

$$
\begin{aligned}
\int_{\Omega_{1}}(\nabla u)^{*} \cdot \nabla v \mathrm{~d} \mathcal{L}^{2 n}+ & \int_{\Omega_{1}}(\nabla u)^{*} \cdot \boldsymbol{X}^{*} \mathrm{~d} \mathcal{L}^{2 n}-\int_{\Omega_{1}} \nabla v \cdot \boldsymbol{X} \mathrm{d} \mathcal{L}^{2 n} \\
& -\int_{\Omega_{1}} \boldsymbol{X} \cdot \boldsymbol{X}^{*} \mathrm{~d} \mathcal{L}^{2 n}=0 .
\end{aligned}
$$


Lemma 3.1 gives

$$
(\nabla u)^{*} \cdot \boldsymbol{X}^{*}=\nabla u \cdot \boldsymbol{X} \quad \text { and } \quad \boldsymbol{X} \cdot \boldsymbol{X}^{*}=0
$$

so that (5.4) becomes

$$
-\int_{\Omega_{1}}(\nabla u)^{*} \cdot(\nabla u-\nabla v) \mathrm{d} \mathcal{L}^{2 n}+\int_{\Omega_{1}} \boldsymbol{X} \cdot(\nabla u-\nabla v) \mathrm{d} \mathcal{L}^{2 n}=0
$$

where we also used the fact that $(\nabla u)^{*} \cdot \nabla u=0$. By the classical Stampacchia theorem, we have $(u-v)^{+} \in W^{1, p}(\Omega)$ and

$$
\nabla(u-v)^{+}=(\nabla(u-v)) \chi_{\Omega_{1}} \quad \text { a.e. in } \Omega,
$$

hence (5.5) can be written as

$$
-\int_{\Omega}(\nabla u)^{*} \cdot \nabla(u-v)^{+} \mathrm{d} \mathcal{L}^{2 n}+\int_{\Omega} \boldsymbol{X} \cdot \nabla(u-v)^{+} \mathrm{d} \mathcal{L}^{2 n}=0 .
$$

Integrating by parts and using the fact that $(u-v)_{\mid \partial \Omega}^{+}=0$ because $u_{\mid \partial \Omega}=v_{\mid \partial \Omega}=\varphi$, we obtain

$$
\begin{aligned}
0 & =-\int_{\Omega}(\nabla u)^{*} \cdot \nabla(u-v)^{+} \mathrm{d} \mathcal{L}^{2 n}+\int_{\Omega} \boldsymbol{X} \cdot \nabla(u-v)^{+} \mathrm{d} \mathcal{L}^{2 n} \\
& =-\int_{\Omega}(\nabla u)^{*} \cdot \nabla(u-v)^{+} \mathrm{d} \mathcal{L}^{2 n}-\int_{\Omega}(u-v)^{+} \operatorname{div} \boldsymbol{X} \mathrm{d} \mathcal{L}^{2 n} \\
& =-\int_{\Omega}(\nabla u)^{*} \cdot \nabla(u-v)^{+} \mathrm{d} \mathcal{L}^{2 n}-2 n \int_{\Omega}(u-v)^{+} \mathrm{d} \mathcal{L}^{2 n} .
\end{aligned}
$$

We claim that

$$
\int_{\Omega}(\nabla u)^{*} \cdot \nabla(u-v)^{+} \mathrm{d} \mathcal{L}^{2 n}=0 .
$$

To this end, consider a sequence $\left(u_{k}\right)_{k \in \mathbb{N}}$ such that

$$
u_{k} \in C^{\infty}(\Omega) \cap W^{1, p^{\prime}}(\Omega) \text { and } \nabla u_{k} \stackrel{*}{\rightarrow} \nabla u \text { in } L^{p^{\prime}}(\Omega) \text { as } k \rightarrow+\infty .
$$

We have also $\left(\nabla u_{k}\right)^{*} \stackrel{*}{\rightarrow} \nabla u^{*}$, thus

$$
\begin{aligned}
\int_{\Omega}(\nabla u)^{*} \cdot \nabla(u-v)^{+} \mathrm{d} \mathcal{L}^{2 n} & =\lim _{k \rightarrow \infty} \int_{\Omega}\left(\nabla u_{k}\right)^{*} \cdot \nabla(u-v)^{+} \mathrm{d} \mathcal{L}^{2 n} \\
& =\int_{\Omega} \operatorname{div}\left((\nabla u)^{*}\right)(u-v)^{+} \mathrm{d} \mathcal{L}^{2 n} \\
& =0
\end{aligned}
$$

by Lemma 3.1 (iii). By (5.6) and (5.7) we deduce that $(u-v)^{+}=0$ a.e. on $\Omega$.

On considering $\Omega_{2}:=\{z \in \Omega: v(z)>u(z)\}$ in place of $\Omega_{1}$, one can similarly prove that $(u-v)^{-}=0$ a.e. on $\Omega$. This completes the proof. 
We introduce now some notations that will be useful also in the proof of the main theorem of the paper. Given a subset $\Omega \subset \mathbb{R}^{2 n}$, a function $u: \Omega \rightarrow \mathbb{R}$, a vector $\tau \in \mathbb{R}^{2 n}$ and $\xi \in \mathbb{R}$ we set

$$
\begin{aligned}
& \Omega_{\tau}:=\left\{z \in \mathbb{R}^{2 n}: z+\tau \in \Omega\right\} \\
& u_{\tau}(z):=u(z+\tau), \quad z \in \Omega_{\tau} \\
& u_{\tau, \xi}^{*}(z):=u_{\tau}(z)+2\left\langle\tau^{*}, z\right\rangle+\xi, \quad z \in \Omega_{\tau} .
\end{aligned}
$$

It is easily seen that, given $\Omega$ open and $u \in \mathrm{BV}(\Omega)$, then both $u_{\tau}$ and $u_{\tau, \xi}^{*}$ belong to $\operatorname{BV}\left(\Omega_{\tau}\right)$. Moreover, if $\Omega$ is bounded with Lipschitz regular boundary one has also

$$
\left(u_{\tau, \xi}^{*}\right)_{\mid \partial\left(\Omega_{\tau}\right)}=\left(u_{\mid \partial \Omega}\right)_{\tau}+2\left\langle\tau^{*}, \cdot\right\rangle+\xi=\left(u_{\mid \partial \Omega}\right)_{\tau, \xi}^{*} \cdot
$$

Remark 5.2. The family of functions $u_{\tau, \xi}^{*}$ has a precise meaning from the viewpoint of Heisenberg groups geometry. Indeed, it is a matter of computations to observe that the $t$-subgraph $E_{u_{\tau, \xi}^{*}}^{t}$ of $u_{\tau, \xi}^{*}$ coincides with the left translation $(-\tau, \xi) \cdot E_{u}^{t}$ (according to the group law) of the $t$-subgraph $E_{u}^{t}$ of $u$ by the element $(-\tau, \xi) \in$ $\mathbb{H}^{n}$.

Lemma 5.3. Let $\Omega \subset \mathbb{R}^{2 n}$ be a bounded open set with Lipschitz regular boundary, $\varphi \in L^{1}(\partial \Omega), \tau \in \mathbb{R}^{2 n}$ and $\xi \in \mathbb{R}$. Then

$$
\mathscr{A}_{\varphi_{\tau, \xi}^{*}, \Omega_{\tau}}\left(u_{\tau, \xi}^{*}\right)=\mathscr{A}_{\varphi, \Omega}(u) \quad \forall u \in \mathrm{BV}(\Omega) .
$$

Proof. Using, e.g., [1, Remark 3.18], it is not difficult to prove that $D u_{\tau}=\ell_{\tau \#}(D u)$, where $\ell_{\tau}$ is the translation $z \mapsto z-\tau$ and $\ell_{\tau \#}$ denotes the push-forward of measures via $\ell_{\tau}$. In particular

$$
\nabla u_{\tau}=(\nabla u)_{\tau}=\nabla u \circ \ell_{\tau}^{-1} \quad \text { and } \quad D^{s} u_{\tau}=\ell_{\tau \#}\left(D^{s} u\right)
$$

hence

$$
D u_{\tau, \xi}^{*}=\left(\nabla u \circ \ell_{\tau}^{-1}+2 \tau^{*}\right) \mathcal{L}^{2 n}+\ell_{\tau \#}\left(D^{s} u\right)
$$

Therefore

$$
\begin{aligned}
& \mathscr{A}_{\varphi_{\tau, \xi}^{*}, \Omega_{\tau}}\left(u_{\tau, \xi}^{*}\right) \\
& =\int_{\Omega_{\tau}}\left|\left(\nabla u \circ \ell_{\tau}^{-1}\right)+2 \tau^{*}+X^{*}\right| \mathrm{d} \mathcal{L}^{2 n}+\left|\ell_{\tau \#}\left(D^{s} u\right)\right|\left(\Omega_{\tau}\right) \\
& \quad+\int_{\partial \Omega_{\tau}}\left|\left(u_{\tau, \xi}^{*}\right)_{\mid \partial\left(\Omega_{\tau}\right)}-\varphi_{\tau, \xi}^{*}\right| \mathrm{d} \mathcal{H}^{2 n-1} .
\end{aligned}
$$

We now use (5.8) and the equality

$$
2 \tau^{*}+\boldsymbol{X}^{*}(z)=2(\tau+z)^{*}=\left(X^{*} \circ \ell_{\tau}^{-1}\right)(z) \quad \forall z \in \mathbb{R}^{2 n}
$$


to get, with a change of variable,

$$
\begin{aligned}
& \mathscr{A}_{\varphi_{\tau, \xi}^{*}, \Omega_{\tau}}\left(u_{\tau, \xi}^{*}\right) \\
& =\int_{\Omega_{\tau}}\left|\nabla u+X^{*}\right| \circ \ell_{\tau}^{-1} \mathrm{~d} \mathcal{L}^{2 n}+\left|\ell_{\tau \#}\left(D^{s} u\right)\right|\left(\ell_{\tau}(\Omega)\right) \\
& \quad+\int_{\partial \Omega_{\tau}}\left|\left(u_{\mid \partial \Omega}-\varphi\right)_{\tau}\right| \mathrm{d} \mathcal{H}^{2 n-1} \\
& =\int_{\Omega}\left|\nabla u+X^{*}\right| \mathrm{d} \mathcal{L}^{2 n}+\left|D^{s} u\right|(\Omega)+\int_{\partial \Omega}\left|u_{\mid \partial \Omega}-\varphi\right| \mathrm{d} \mathcal{H}^{2 n-1} \\
& =\mathscr{A}_{\varphi, \Omega}(u) .
\end{aligned}
$$

Corollary 5.4. Under the same assumptions of Lemma 5.3: if $\bar{u}$ and $\underline{u}$ are as in Proposition 4.4 , then $(\bar{u})_{\tau, \xi}^{*},(\underline{u})_{\tau, \xi}^{*} \in \mathscr{M}_{\varphi_{\tau, \xi}^{*}}$ and

$$
(\bar{u})_{\tau, \xi}^{*} \leq u \leq(\underline{u})_{\tau, \xi}^{*} \quad \mathcal{L}^{2 n} \text {-a.e. in } \Omega_{\tau}, \forall u \in \mathscr{M}_{\varphi_{\tau, \xi}^{*}} .
$$

The next theorem states that, whenever we fix an affine boundary datum, the functional with the penalization on the boundary has a unique minimizer that is the affine function itself. This is one of the main tools in the proof of Theorem 1.1.

Theorem 5.5. Let $\Omega \subset \mathbb{R}^{2 n}$ be a bounded open set with Lipschitz regular boundary and let $L: \mathbb{R}^{2 n} \rightarrow \mathbb{R}$ be an affine function, i.e., $L(z)=\langle a, z\rangle+b$ for some $a \in \mathbb{R}^{2 n}, b \in \mathbb{R}$. Then $L$ is the unique solution of

$$
\min \left\{\mathscr{A}_{L, \Omega}(u): u \in \mathrm{BV}(\Omega)\right\} .
$$

Proof. We divide the proof into several steps.

Step 1. reduction to the case $L=0$.

Setting $\tau:=a^{*} / 2 \in \mathbb{R}^{2 n}$ and $\xi=-b$, one has $L_{\tau, \xi}^{*} \equiv 0$. By Lemma 5.3 and Corollary 5.4, the fact that $L$ is the unique solution of $(5.9)$ is equivalent to the fact that 0 is the unique minimizer of the problem

$$
\min \left\{\mathscr{A}_{0, \Omega_{\tau}}(u): u \in \mathrm{BV}\left(\Omega_{\tau}\right)\right\} .
$$

In view of this, we can henceforth assume that $L=0$.

Step 2. $L=0$ is a minimizer for (5.9).

Let $u \in \mathrm{BV}(\Omega)$; by the dominated convergence theorem we have

$$
\begin{aligned}
& \int_{\Omega}\left\langle\sigma_{u}, \frac{\boldsymbol{X}^{*}}{\left|\boldsymbol{X}^{*}\right|}\right\rangle \mathrm{d}|D u|=\lim _{\epsilon \rightarrow 0} \int_{\Omega}\left\langle\sigma_{u}, \frac{\boldsymbol{X}^{*}}{\left|\boldsymbol{X}^{*}\right|+\epsilon}\right\rangle \mathrm{d}|D u| \\
& =\lim _{\epsilon \rightarrow 0}\left[-\int_{\Omega} u \operatorname{div}\left(\frac{\boldsymbol{X}^{*}}{\left|\boldsymbol{X}^{*}\right|+\epsilon}\right) \mathrm{d} \mathcal{L}^{2 n}+\int_{\partial \Omega} u \mid \partial \Omega\left\langle v_{\Omega}, \frac{\boldsymbol{X}^{*}}{\left|\boldsymbol{X}^{*}\right|+\epsilon}\right\rangle \mathrm{d} \mathcal{H}^{2 n-1}\right] \\
& =\int_{\partial \Omega} u_{\mid \partial \Omega}\left\langle v_{\Omega}, \frac{\boldsymbol{X}^{*}}{\left|\boldsymbol{X}^{*}\right|}\right\rangle \mathrm{d} \mathcal{H}^{2 n-1},
\end{aligned}
$$


where we have used the fact that $\operatorname{div}\left(\frac{\boldsymbol{X}^{*}}{\left|\boldsymbol{X}^{*}\right|+\epsilon}\right)=0$. Thus

$$
\begin{aligned}
& \mathscr{A}_{0, \Omega}(u)=\int_{\Omega}\left|\nabla u+\boldsymbol{X}^{*}\right| \mathrm{d} \mathcal{L}^{2 n}+\left|D^{s} u\right|(\Omega)+\int_{\partial \Omega}\left|u_{\mid \partial \Omega}\right| \mathrm{d} \mathcal{H}^{2 n-1} \\
& \geq \int_{\Omega}\left\langle\nabla u+\boldsymbol{X}^{*}, \frac{\boldsymbol{X}^{*}}{\left|\boldsymbol{X}^{*}\right|}\right\rangle \mathrm{d} \mathcal{L}^{2 n}+\int_{\Omega}\left\langle\sigma_{u}, \frac{\boldsymbol{X}^{*}}{\left|\boldsymbol{X}^{*}\right|}\right\rangle \mathrm{d}\left|D^{s} u\right|+\int_{\partial \Omega}\left|u_{\mid \partial \Omega}\right| \mathrm{d} \mathcal{H}^{2 n-1} \\
& =\int_{\Omega}\left\langle\sigma_{u}, \frac{\boldsymbol{X}^{*}}{\left|\boldsymbol{X}^{*}\right|}\right\rangle \mathrm{d}|D u|+\int_{\Omega}\left\langle\boldsymbol{X}^{*}, \frac{\boldsymbol{X}^{*}}{\left|\boldsymbol{X}^{*}\right|}\right\rangle \mathrm{d} \mathcal{L}^{2 n}+\int_{\partial \Omega}\left|u_{\mid \partial \Omega}\right| \mathrm{d} \mathcal{H}^{2 n-1} \\
& =\int_{\Omega}\left|\boldsymbol{X}^{*}\right| \mathcal{L}^{2 n}+\int_{\partial \Omega}\left|u_{\mid \partial \Omega}\right|\left(1+\operatorname{sgn}\left(u_{\mid \partial \Omega}\right)\left\langle v_{\Omega}, \frac{\boldsymbol{X}^{*}}{\left|\boldsymbol{X}^{*}\right|}\right\rangle\right) \mathrm{d} \mathcal{H}^{2 n-1} \\
& \geq \int_{\Omega}\left|\boldsymbol{X}^{*}\right| \mathcal{L}^{2 n}=\mathscr{A}_{0, \Omega}(0),
\end{aligned}
$$

which proves that $L=0$ is a minimizer of (5.9).

Step 3. if $\Omega=B(0, R)$ for some $R>0$, then $L=0$ is the unique minimizer of (5.9).

Since $\Omega$ is a ball centered at the origin, we have $\left\langle v_{\Omega}, \frac{X^{*}}{\left|X^{*}\right|}\right\rangle=0$ and, discarding its third line, (5.10) can be rewritten as

$$
\begin{aligned}
& \mathscr{A}_{0, \Omega}(u)=\int_{\Omega}\left|\nabla u+\boldsymbol{X}^{*}\right| \mathrm{d} \mathcal{L}^{2 n}+\left|D^{s} u\right|(\Omega)+\int_{\partial \Omega}|u| \partial \Omega \mid \mathrm{d} \mathcal{H}^{2 n-1} \\
& \geq \int_{\Omega}\left\langle\nabla u+\boldsymbol{X}^{*}, \frac{\boldsymbol{X}^{*}}{\left|\boldsymbol{X}^{*}\right|}\right\rangle \mathrm{d} \mathcal{L}^{2 n}+\int_{\Omega}\left\langle\sigma_{u}, \frac{\boldsymbol{X}^{*}}{\left|\boldsymbol{X}^{*}\right|}\right\rangle \mathrm{d}\left|D^{s} u\right|+\int_{\partial \Omega}\left|u_{\mid \partial \Omega}\right| \mathrm{d} \mathcal{H}^{2 n-1} \\
& =\int_{\Omega}\left|\boldsymbol{X}^{*}\right| \mathcal{L}^{2 n}+\int_{\partial \Omega}\left|u_{\mid \partial \Omega}\right| \mathrm{d} \mathcal{H}^{2 n-1} \\
& \geq \int_{\Omega}\left|\boldsymbol{X}^{*}\right| \mathcal{L}^{2 n}=\mathscr{A}_{0, \Omega}(0) .
\end{aligned}
$$

Let $u \in \mathrm{BV}(\Omega)$ be a minimizer for (5.9); then (by Step 2) $\mathscr{A}_{0, \Omega}(u)=\mathscr{A}_{0, \Omega}(0)$ and the two inequalities in (5.11) must be equalities. In particular, one has $u_{\mid \partial \Omega}=0$ and

$$
\begin{aligned}
& \int_{\Omega}\left|\nabla u+X^{*}\right| \mathrm{d} \mathcal{L}^{2 n}=\int_{\Omega}\left\langle\nabla u+X^{*}, \frac{X^{*}}{\left|\boldsymbol{X}^{*}\right|}\right\rangle \mathrm{d} \mathcal{L}^{2 n}, \\
& \left|D^{s} u\right|(\Omega)=\int_{\Omega}\left\langle\sigma_{u}, \sigma_{u}\right\rangle \mathrm{d}\left|D^{s} u\right|=\int_{\Omega}\left\langle\sigma_{u}, \frac{\boldsymbol{X}^{*}}{\left|\boldsymbol{X}^{*}\right|}\right\rangle \mathrm{d}\left|D^{s} u\right|,
\end{aligned}
$$

so that $\sigma_{u}=\frac{X^{*}}{\left|X^{*}\right|}\left|D^{s} u\right|$-a.e. and there exists a measurable function $\lambda: \Omega \rightarrow$ $[0,+\infty)$ such that

$$
\sigma_{u}|\nabla u|+\boldsymbol{X}^{*}=\nabla u+\boldsymbol{X}^{*}=\lambda \frac{\boldsymbol{X}^{*}}{\left|\boldsymbol{X}^{*}\right|} \quad \mathcal{L}^{2 n} \text {-a.e on } \Omega .
$$


All in all, there exists a $|D u|$-measurable function $\tilde{\lambda}: \Omega \rightarrow \mathbb{R}$ such that

$$
\sigma_{u}=\tilde{\lambda} \boldsymbol{X}^{*} \quad|D u| \text {-a.e. in } \Omega .
$$

We claim that, up to the choice of a representative, any function $u$ satisfying (5.12) is 0-homogeneous, i.e., it satisfies $u(z)=u(t z)$ for any $z \in B(0, R)$ and $t \in$ $(0,1)$; roughly speaking, (5.12) says indeed that $u$ has null radial derivative, which suggests its 0 -homogeneity. Recalling that $u_{\mid \partial \Omega}=0$, this would be enough to conclude that $u \equiv 0$.

Consider the map

$$
\begin{gathered}
F:[0, R) \times \mathbb{S}^{2 n-1} \rightarrow \Omega=B(0, R) \\
(\rho, \theta) \longmapsto \rho \theta .
\end{gathered}
$$

The claimed 0-homogeneity of $u$ is clearly equivalent to the fact that $u_{0}:=u \circ F$ : $[0, R] \times \mathbb{S}^{2 n-1} \rightarrow \mathbb{R}$ admits a representative which does not depend on $\rho$. Thus, it will be enough to prove that for any $f_{0} \in C_{c}^{\infty}\left((0, R) \times \mathbb{S}^{2 n-1}\right)$ there holds

$$
\int_{(0, R) \times \mathbb{S}^{2 n-1}} u_{0} \frac{\partial f_{0}}{\partial \rho} \mathrm{d}\left(\mathcal{L}^{1} \otimes \mu\right)=0
$$

where $\mu$ is the Haar measure on $\mathbb{S}^{2 n-1}$. Define $f \in C_{c}^{\infty}(B(0, R) \backslash\{0\})$ by $f(z):=$ $\left(f_{0} \circ F^{-1}\right)(z)=f_{0}\left(|z|, \frac{z}{|z|}\right)$ for any $z \in B(0, R) \backslash\{0\}$; notice that

$$
\left(\left(\frac{\partial f_{0}}{\partial \rho}\right) \circ F^{-1}\right)(z)=\frac{\partial f_{0}}{\partial \rho}\left(|z|, \frac{z}{|z|}\right)=\left\langle\nabla f(z), \frac{z}{|z|}\right\rangle .
$$

By a change of variable we get

$$
\begin{aligned}
& \int_{(0, R) \times \mathbb{S}^{2 n-1}} u_{0} \frac{\partial f_{0}}{\partial \rho} \mathrm{d}\left(\mathcal{L}^{1} \otimes \mu\right) \\
& =\int_{(0, R) \times \mathbb{S}^{2 n-1}} u(F(\rho, \theta))\left(\left(\frac{\partial f_{0}}{\partial \rho}\right) \circ F^{-1}\right)(F(\rho, \theta)) \frac{1}{\rho^{2 n-1}} \rho^{2 n-1} \mathrm{~d}\left(\mathcal{L}^{1} \otimes \mu\right)(\rho, \theta) \\
& =\int_{B(0, R)} u(z)\left\langle\nabla f(z), \frac{z}{|z|}\right\rangle \frac{1}{|z|^{2 n-1}} \mathrm{~d} \mathcal{L}^{2 n}(z) \\
& =\sum_{i=1}^{2 n} \int_{B(0, R)} u(z) \frac{z_{i}}{|z|^{2 n}} \frac{\partial f}{\partial z_{i}}(z) \mathrm{d} \mathcal{L}^{2 n}(z) \\
& =-\int_{B(0, R)} u(z) f(z)\left[\sum_{i=1}^{2 n} \frac{\partial}{\partial z_{i}}\left(\frac{z_{i}}{|z|^{2 n}}\right)\right] \mathrm{d} \mathcal{L}^{2 n}(z) \\
& \quad-\int_{B(0, R)} f(z)\left\langle\sigma_{u}(z), z\right\rangle \frac{1}{|z|^{2 n}} \mathrm{~d}|D u| .
\end{aligned}
$$


Our claim (5.13) is then a consequence of the equality

$$
\sum_{i=1}^{2 n} \frac{\partial}{\partial z_{i}}\left(\frac{z_{i}}{|z|^{2 n}}\right)=0
$$

and the fact that $\sigma_{u}(z)=\left(\tilde{\lambda} X^{*}\right)(z)$ is $|D u|$-a.e. orthogonal to $z$.

Step 4. $L=0$ is the unique minimizer of (5.9) for general $\Omega$.

Let $u \in \operatorname{BV}(\Omega)$ be a minimizer of (5.9) and let $R>0$ be such that $\Omega \Subset$ $B(0, R)$. Let us define

$$
u_{0}(z):= \begin{cases}u(z) & \text { if } z \in \Omega \\ 0 & \text { if } z \in B(0, R) \backslash \bar{\Omega}\end{cases}
$$

By Step 2, also $L=0$ is a minimizer, i.e., $\mathscr{A}_{0, \Omega}(u)=\mathscr{A}_{\Omega}(0)$; thus

$$
\begin{aligned}
\mathscr{A}_{0, B(0, R)}\left(u_{0}\right)= & \int_{\Omega} \mid \nabla u \\
& +X^{*}\left|\mathrm{~d} \mathcal{L}^{2 n}+\right| D^{s} u\left|(\Omega)+\int_{B(0, R) \backslash \bar{\Omega}}\right| X^{*}\left|\mathrm{~d} \mathcal{L}^{2}+\right| D^{s} u_{0} \mid(\partial \Omega) \\
= & \mathscr{A}_{\Omega}(u)+\mathscr{A}_{B(0, R) \backslash \bar{\Omega}}(0)+\int_{\partial \Omega}|u| \partial \Omega \mid \mathrm{d} \mathcal{H}^{2 n-1} \\
= & \mathscr{A}_{0, \Omega}(u)+\mathscr{A}_{B(0, R) \backslash \bar{\Omega}}(0) \\
= & \mathscr{A}_{\Omega}(0)+\mathscr{A}_{B(0, R) \backslash \bar{\Omega}}(0)=\mathscr{A}_{B(0, R)}(0)=\mathscr{A}_{0, B(0, R)}(0) .
\end{aligned}
$$

Therefore, $u_{0}$ is a minimizer of $\mathscr{A}_{0, B(0, R)}$; by Step 3, this implies that $u_{0}=0$, i.e., that $u=0 \mathcal{L}^{2 n}$-a.e. on $\Omega$, as desired.

The next corollary is a special comparison principle for affine functions, and in particular shows that affine functions satisfy a comparison principle both from above and from below.

Corollary 5.6. Let $\Omega \subset \mathbb{R}^{2 n}$ be a bounded open set with Lipschitz boundary, $\varphi \in$ $L^{1}(\partial \Omega)$ and $L: \mathbb{R}^{2 n} \rightarrow \mathbb{R}$ be an affine function, i.e., $L(z)=\langle a, z\rangle+b$ for some $a \in \mathbb{R}^{2 n}, b \in \mathbb{R}$.

i) Assume that $\varphi \leq L \mathcal{H}^{2 n-1}$-a.e. on $\partial \Omega$. Then, for any minimizer $u \in \mathscr{M}_{\varphi}$ of $\mathscr{A}_{\varphi}$, we have $u \leq L \mathcal{L}^{2 n}$-a.e. in $\Omega$.

ii) Assume that that $\varphi \geq L \mathcal{H}^{2 n-1}$-a.e. on $\partial \Omega$. Then, for any minimizer $u \in \mathscr{M}_{\varphi}$ of $\mathscr{A}_{\varphi}$, we have $u \geq L \mathcal{L}^{2 n}$-a.e. in $\Omega$.

Proof. Both claims follow immediately from Theorem 4.5 when we observe that the set $\mathscr{M}_{L}$ consists of just one element that is $L$ itself, so that, following the notations of Proposition $4.4, L=\bar{L}=\underline{L}$. 


\section{The Bounded Slope Condition}

We recall the well-known definition of Bounded Slope Condition (see [32]) for boundary data. In particular we refer to [27] also for some classical results that we will summarize next.

Definition 6.1. We say that a function $\varphi: \partial \Omega \rightarrow \mathbb{R}$ satisfies the bounded slope condition with constant $Q>0$ ( $Q$-B.S.C. for short, or simply B.S.C. when the constant $Q$ does not play any role) if for every $z_{0} \in \partial \Omega$ there exist two affine functions $w_{z_{0}}^{+}$and $w_{z_{0}}^{-}$such that

$$
\begin{aligned}
& w_{z_{0}}^{-}(z) \leq \varphi(z) \leq w_{z_{0}}^{+}(z) \quad \forall z \in \partial \Omega, \\
& w_{z_{0}}^{-}\left(z_{0}\right)=\varphi\left(z_{0}\right)=w_{z_{0}}^{+}\left(z_{0}\right) \\
& \operatorname{Lip}\left(w_{z_{0}}^{-}\right) \leq Q \quad \text { and } \quad \operatorname{Lip}\left(w_{z_{0}}^{+}\right) \leq Q
\end{aligned}
$$

where $\operatorname{Lip}(w)$ denotes the Lipschitz constant of $w$.

We also recall that a set $\Omega \subset \mathbb{R}^{2 n}$ is said to be uniformly convex if there exists a positive constant $C=C(\Omega)$ and, for each $z_{0} \in \partial \Omega$, a hyperplane $\Pi_{z_{0}}$ passing through $z_{0}$ such that

$$
\left|z-z_{0}\right|^{2} \leq C \operatorname{dist}\left(z, \Pi_{z_{0}}\right) \quad \forall z \in \partial \Omega,
$$

where $\operatorname{dist}(z, \Omega):=\inf \{|z-w| \mid w \in \Omega\}$. It is worth noticing that, if $\partial \Omega$ is of class $C^{2}$, this condition holds if and only if all principal curvatures of $\partial \Omega$ are strictly positive, see [27] for details.

Remark 6.2. We collect here some facts on the B.S.C.

a) If $\varphi: \partial \Omega \rightarrow \mathbb{R}$ satisfies the B.S.C. and is not affine, then $\Omega$ has to be convex (see [27, page 20]) and $\varphi$ is Lipschitz continuous on $\partial \Omega$. Moreover, if $\partial \Omega$ has flat faces, then $\varphi$ has to be affine on them.

This property seems to say that the B.S.C. is a quite restrictive assumption. Anyhow the following one, due to M. Miranda [43] (see also [27, Theorem 1.1]), shows that the class of functions satisfying the B.S.C. on a uniformly convex set is quite large.

b) Let $\Omega \subset \mathbb{R}^{n}$ be open, bounded and uniformly convex; then every $\varphi \in C^{1,1}\left(\mathbb{R}^{n}\right)$ satisfies the B.S.C. on $\partial \Omega$.

We denote by $f, g$ the functions defined, respectively, by $f(z):=\sup _{z_{0} \in \partial \Omega} w_{z_{0}}^{-}(z)$ and $g(z):=\inf _{z_{0} \in \partial \Omega} w_{z_{0}}^{+}(z)$. We underline that $f$ is a convex function, $g$ is a concave function and both are Lipschitz with Lipschitz constant not greater than $Q$. 
Lemma 6.3. Let $\Omega \subset \mathbb{R}^{2 n}$ be an open bounded set with Lipschitz regular boundary; assume that $\varphi \in L^{1}(\partial \Omega)$ satisfies the $Q-B . S . C$. Then, for any $u \in \mathscr{M}_{\varphi}$ there holds

i) $u_{\mid \partial \Omega}=\varphi$;

ii) $f \leq u \leq g \mathcal{L}^{2 n}$-a.e. in $\Omega$;

iii) $u$ is also a minimizer of $\mathscr{A}_{\Omega}$ in $\mathrm{BV}(\Omega)$ with $u_{\mid \partial \Omega}=\varphi$.

Proof. i) For every $z_{0} \in \partial \Omega$, let $w_{z_{0}}^{+}$and $w_{z_{0}}^{-}$be as in Definition 6.1. By Corollary 5.6, we have that $w_{z_{0}}^{-} \leq u \leq w_{z_{0}}^{+} \mathcal{L}^{2 n}$-a.e. in $\Omega$. Recalling (6.1) we obtain

$$
\left|u(z)-\varphi\left(z_{0}\right)\right| \leq Q\left|z-z_{0}\right| \quad \mathcal{L}^{2 n} \text {-a.e. } z \in \Omega, \forall z_{0} \in \partial \Omega .
$$

Therefore,

$$
\frac{1}{\rho^{2 n}} \int_{\Omega \cap B\left(z_{0}, \rho\right)}\left|u-\varphi\left(z_{0}\right)\right| \mathrm{d} \mathcal{L}^{2 n} \leq \frac{Q}{\rho^{2 n}} \int_{\Omega \cap B\left(z_{0}, \rho\right)}\left|z-z_{0}\right| \mathrm{d} \mathcal{L}^{2 n}(z) \leq Q \rho
$$

and letting $\rho \rightarrow 0^{+}$in (6.3) we conclude that $u_{\mid \partial \Omega}=\varphi$.

ii) Fix a Lebsgue point $\bar{z} \in \Omega$ of $u$. Since $f$ is a convex function, there exists $\xi \in \mathbb{R}^{2 n}$ such that $f(z) \geq f(\bar{z})+\xi \cdot(z-\bar{z}):=h(z)$ for every $z \in \bar{\Omega}$. The function $h$ is affine and $h \leq \varphi$ on $\partial \Omega$; then Corollary 5.6 implies that $u \geq h \mathcal{L}^{2 n}$-a.e. in $\Omega$. Considering the mean integral on a ball centered at $\bar{z}$ we obtain

$$
f_{B(\bar{z}, \rho)} u(z) d \mathcal{L}^{2 n} \geq f_{B(\bar{z}, \rho)} h(z) d \mathcal{L}^{2 n}
$$

and, passing to the limit as $\rho \rightarrow 0^{+}$, we get $u(\bar{z}) \geq f(\bar{z})$. One can argue in a similar way to prove that $u \leq g \mathcal{L}^{2 n}$-a.e. in $\Omega$.

Finally, the proof of iii) is straightforward.

Remark 6.4. If $\Omega^{\prime} \subset \Omega$ are open bounded domains with Lipschitz regular boundary and $u \in \mathrm{BV}(\Omega)$, we use the notation $\mathscr{A}_{u, \Omega^{\prime}}$ to denote the functional $\mathscr{A}_{u_{\mid \partial \Omega^{\prime}}, \Omega^{\prime}}$. Let us prove that, if $u$ is a minimizer of $\mathscr{A}_{\varphi, \Omega}$ with $\varphi=u_{\mid \partial \Omega}$, then $u$ is also a minimizer of $\mathscr{A}_{u, \Omega^{\prime}}$.

Let us write $\Gamma:=\partial \Omega^{\prime} \cap \Omega$ and $\partial \Omega=\Delta_{1} \cup \Delta_{2}$, where

$$
\Delta_{1}:=\partial \Omega \cap \partial \Omega^{\prime} \quad \text { and } \quad \Delta_{2}:=\partial \Omega \backslash \partial \Omega^{\prime} .
$$


Notice that $\partial \Omega^{\prime}=\Gamma \cup \Delta_{1}$. We also denote by $u_{i}, u_{o}: \Gamma \rightarrow \mathbb{R}$ the "inner" and "outer" (with respect to $\Omega^{\prime}$ ) traces of $u$ on $\Gamma$, i.e.,

$$
u_{i}:=\left(u_{\mid \partial \Omega^{\prime}}\right)\left\llcorner\Gamma \quad \text { and } \quad u_{o}:=\left(u_{\mid \partial\left(\Omega \backslash \overline{\Omega^{\prime}}\right)}\right)\llcorner\Gamma .\right.
$$

Assume by contradiction that $u$ is not a minimizer of $\mathscr{A}_{u, \Omega^{\prime}}$; then, there exists $v \in \mathrm{BV}\left(\Omega^{\prime}\right)$ such that

$$
\begin{aligned}
0< & \mathscr{A}_{u, \Omega^{\prime}}(u)-\mathscr{A}_{u, \Omega^{\prime}}(v) \\
= & \mathscr{A}_{\Omega^{\prime}}(u)-\mathscr{A}_{\Omega^{\prime}}(v)-\int_{\partial \Omega^{\prime}}\left|v_{\mid \partial \Omega^{\prime}}-u_{\mid \partial \Omega^{\prime}}\right| \mathrm{d} \mathcal{H}^{2 n-1} \\
= & \mathscr{A}_{\Omega^{\prime}}(u)-\mathscr{A}_{\Omega^{\prime}}(v)-\int_{\Gamma}\left|v_{\mid \partial \Omega^{\prime}}-u_{i}\right| \mathrm{d} \mathcal{H}^{2 n-1} \\
& -\int_{\Delta_{1}}\left|v_{\mid \partial \Omega^{\prime}}-\varphi\right| \mathrm{d} \mathcal{H}^{2 n-1} .
\end{aligned}
$$

We will reach a contradiction if we show that the function $w \in \mathrm{BV}(\Omega)$ defined by

$$
w:=v \text { on } \Omega^{\prime}, \quad w:=u \text { on } \Omega \backslash \Omega^{\prime}
$$

satisfies $\mathscr{A}_{\varphi, \Omega}(u)-\mathscr{A}_{\varphi, \Omega}(w)>0$.

Let us compute

$$
\begin{aligned}
\mathscr{A}_{\varphi, \Omega}(u) & =\mathscr{A}_{\Omega}(u)=\mathscr{A}_{\Omega^{\prime}}(u)+\mathscr{A}_{\Omega \backslash \overline{\Omega^{\prime}}}(u)+\left|D^{s} u\right|(\Gamma) \\
& =\mathscr{A}_{\Omega^{\prime}}(u)+\mathscr{A}_{\Omega \backslash \overline{\Omega^{\prime}}}(u)+\int_{\Gamma}\left|u_{o}-u_{i}\right| \mathrm{d} \mathcal{H}^{2 n-1}
\end{aligned}
$$

(we have used the assumption $\varphi=u_{\mid \partial \Omega}$ ) and

$$
\begin{aligned}
\mathscr{A}_{\varphi, \Omega}(v)= & \mathscr{A}_{\Omega^{\prime}}(v)+\mathscr{A}_{\Omega \backslash \overline{\Omega^{\prime}}}(u)+\left|D^{s} w\right|(\Gamma)+\int_{\partial \Omega}\left|w_{\mid \partial \Omega}-\varphi\right| \mathrm{d} \mathcal{H}^{2 n-1} \\
= & \mathscr{A}_{\Omega^{\prime}}(v)+\mathscr{A}_{\Omega \backslash \overline{\Omega^{\prime}}}(u)+\int_{\Gamma}\left|v_{\mid \partial \Omega^{\prime}}-u_{o}\right| \mathrm{d} \mathcal{H}^{2 n-1} \\
& +\int_{\Delta_{1}}\left|v_{\mid \partial \Omega}-\varphi\right| \mathrm{d} \mathcal{H}^{2 n-1} .
\end{aligned}
$$

Therefore

$$
\begin{aligned}
& \mathscr{A}_{\varphi, \Omega}(u)-\mathscr{A}_{\varphi, \Omega}(v) \\
& =\mathscr{A}_{\Omega^{\prime}}(u)-\mathscr{A}_{\Omega^{\prime}}(v)+\int_{\Gamma}\left(\left|u_{o}-u_{i}\right|-\left|v_{\mid \partial \Omega^{\prime}}-u_{o}\right|\right) \mathrm{d} \mathcal{H}^{2 n-1}-\int_{\Delta_{1}}\left|v_{\mid \partial \Omega}-\varphi\right| \mathrm{d} \mathcal{H}^{2 n-1} \\
& \geq \mathscr{A}_{\Omega^{\prime}}(u)-\mathscr{A}_{\Omega^{\prime}}(v)-\int_{\Gamma}\left|v_{\mid \partial \Omega^{\prime}}-u_{i}\right| \mathrm{d} \mathcal{H}^{2 n-1}-\int_{\Delta_{1}}\left|v_{\mid \partial \Omega^{\prime}}-\varphi\right| \mathrm{d} \mathcal{H}^{2 n-1} \\
& >0
\end{aligned}
$$

by (6.4), as desired. 
We are now in position to prove our main result.

Proof of Theorem 1.1. We divide the proof into several steps.

Step 1 . We denote by $\bar{u}$ the (pointwise a.e.) maximum of the minimizers of $\mathscr{A}_{\varphi, \Omega}$ in BV (see Theorem 4.3). Lemma 6.3 implies that $f \leq \bar{u} \leq g \mathcal{L}^{2 n}$-a.e. in $\Omega$ and $\bar{u}=\varphi=f=g$ on $\partial \Omega$; in particular, $\bar{u}$ is also a minimizer for (1.1).

Let $\tau \in \mathbb{R}^{2 n}$ be such that $\Omega \cap \Omega_{\tau} \neq \emptyset$; following the notations introduced in Section 5 , we consider the function $\bar{u}_{\tau, 0}^{*}$, which we denote by $\bar{u}_{\tau}^{*}$ to simplify the notation. Let us consider the set $\Omega \cap \Omega_{\tau}$. By Remark 6.4, $\bar{u}$ is a minimizer of $\mathscr{A}_{\bar{u}, \Omega \cap \Omega_{\tau}}$ and, by Corollary 5.4 and Remark 6.4, $\bar{u}_{\tau}^{*}$ is a minimizer of $\mathscr{A}_{\bar{u}_{\tau}^{*}, \Omega \cap \Omega_{\tau}}$. Let $z \in \partial\left(\Omega \cap \Omega_{\tau}\right)$, then either $z \in \partial \Omega$ or $z \in \partial \Omega_{\tau}$.

If $z \in \partial \Omega$, then $z+\tau \in \bar{\Omega}$ and the inequality (6.2) in Lemma 6.3 implies that

$$
\bar{u}(z)-Q|\tau| \leq \bar{u}(z+\tau) \leq \bar{u}(z)+Q|\tau| .
$$

Otherwise, $z \in \partial \Omega_{\tau}$ and $z=(z+\tau)-\tau \in \bar{\Omega}$, and Lemma 6.3 implies again (6.5).

So we have proved that (6.5) holds for any $z \in \partial\left(\Omega \cap \Omega_{\tau}\right)$, hence

$$
\bar{u}(z)-Q|\tau|+2\left\langle\tau^{*}, z\right\rangle \leq \bar{u}(z+\tau)+2\left\langle\tau^{*}, z\right\rangle \leq \bar{u}(z)+Q|\tau|+2\left\langle\tau^{*}, z\right\rangle .
$$

Setting $M:=Q+2 \sup _{z \in \Omega}|z|$, one has

$$
\bar{u}(z)-M|\tau| \leq \bar{u}_{\tau}^{*}(z) \leq \bar{u}(z)+M|\tau| \quad \text { for any } z \in \partial\left(\Omega \cap \Omega_{\tau}\right)
$$

and, by Corollary 4.6,

$$
\bar{u}(z)-M|\tau| \leq \bar{u}_{\tau}^{*}(z) \leq \bar{u}(z)+M|\tau| \quad \text { for } \mathcal{L}^{2 n} \text {-a.e. } z \in \Omega \cap \Omega_{\tau} .
$$

This is equivalent to

$\bar{u}(z)-M|\tau|-2\left\langle\tau^{*}, z\right\rangle \leq \bar{u}(z+\tau) \leq \bar{u}(z)+M|\tau|-2\left\langle\tau^{*}, z\right\rangle$ for $\mathcal{L}^{2 n}$-a.e. $z \in \Omega \cap \Omega_{\tau}$ and, setting $K:=M+2 \sup _{z \in \Omega}|z|$,

$$
\bar{u}(z)-K|\tau| \leq \bar{u}(z+\tau) \leq \bar{u}(z)+K|\tau| \quad \text { for } \mathcal{L}^{2 n} \text {-a.e. } z \in \Omega \cap \Omega_{\tau} .
$$

Step 2. We claim that the inequality $|\bar{u}(z)-\bar{u}(\bar{z})| \leq K|z-\bar{z}|$ holds for any Lebesgue points $z, \bar{z}$ of $\bar{u}$. We define $\tau:=\bar{z}-z$; then $\Omega \cap \Omega_{\tau} \neq \emptyset$ and, arguing as in Step 1, we obtain

$$
\left|\bar{u}\left(z^{\prime}+\tau\right)-\bar{u}\left(z^{\prime}\right)\right| \leq K|\tau| \text { for } \mathcal{L}^{2 n} \text {-a.e. } z^{\prime} \in \Omega \cap \Omega_{\tau} .
$$

Let $\rho>0$ be such that $B(z, \rho) \subset \Omega \cap \Omega_{\tau}$ and $B(\bar{z}, \rho) \subset \Omega \cap \Omega_{\tau}$; then

$$
\begin{aligned}
|\bar{u}(z)-\bar{u}(\bar{z})| & =\left|\lim _{\rho \rightarrow 0}\left(f_{B(z, \rho)} \bar{u}\left(z^{\prime}\right) d z^{\prime}-f_{B(\bar{z}, \rho)} \bar{u}\left(z^{\prime}\right) d z^{\prime}\right)\right| \\
& \leq \lim _{\rho \rightarrow 0} f_{B(z, \rho)}\left|\bar{u}\left(z^{\prime}\right)-\bar{u}\left(z^{\prime}+\tau\right)\right| d z^{\prime} \leq K|z-\bar{z}| .
\end{aligned}
$$


Step 3. We have proved that $\bar{u}$, the maximum of the minimizer of $\mathscr{A}_{\varphi}$, has a representative that is Lipschitz continuous on $\Omega$, with Lipschitz constant not greater than $K=Q+4 \sup _{z \in \Omega}|z|$. The same argument leads to prove that $\underline{u}$, the minimum of the minimizers of $\mathscr{A}_{\varphi}$, has a representative that is Lipschitz continuous on $\Omega$, with Lipschitz constant not greater than $K$. The uniqueness criterion in Proposition 5.1 (with $p=1$ ) implies that $\bar{u}=\underline{u} \mathcal{L}^{2 n}$-a.e. on $\Omega$. If $u$ is another minimizer of $\mathscr{A}_{\varphi}$, we have by Proposition 4.4 that $\underline{u} \leq u \leq \bar{u} \mathcal{L}^{2 n}$-a.e. on $\Omega$. This concludes the proof.

Remark 6.5. The bound $Q+4 \sup _{z \in \Omega}|z|$ on the Lipschitz constant of the minimizer might not be optimal. Actually, we do not even know whether, in contrast with the classical case (see [27, Theorem 1.2]), there are examples of minimizers of $\mathscr{A}$ satisfying the $Q$-B.S.C. whose Lipschitz constant is larger than $Q$.

The following examples show that, at least in the case $n=1$, Theorem 1.1 is sharp, in the sense that minimizers might not be better than Lipschitz regular.

Example 6.6. It was proved ${ }^{2}$ in [14, Example 7.2] that the Lipschitz function

$$
u(x, y):= \begin{cases}2 x y & \text { if } y>0 \\ 0 & \text { if } y \leq 0\end{cases}
$$

is a minimizer of $\mathscr{A}_{u_{\mid \partial \Omega}, \Omega}$ on any bounded open set $\Omega$ with Lipschitz regular boundary. Let us prove that $u \mid \partial \Omega$ satisfies the B.S.C. on the open set

$$
\Omega:=\left\{(x, y) \in \mathbb{R}^{2}: x^{2}-1<y<1-x^{2}\right\} .
$$

Upon setting $\varphi(x, y):=x\left(y-x^{2}+1\right)$, one can easily check that $u(x, y)=\varphi(x, y)$ for any $(x, y) \in \partial \Omega$; moreover, $\Omega$ is uniformly convex and $\varphi \in C^{\infty}\left(\mathbb{R}^{2}\right)$, thus $u_{\mid \partial \Omega}$ satisfies the B.S.C. on $\Omega$ because of Remark 6.2 (b). By Theorem 1.1, $u$ is the unique minimizer of $\mathscr{A}_{\varphi, \Omega}$ on $\mathrm{BV}(\Omega)$; notice that $u$ is not better than Lipschitz continuous on $\Omega$.

Example 6.7. The previous example provides a nonsmooth minimizer of $\mathscr{A}$ on a nonsmooth domain; it is anyway possible to exhibit nonsmooth minimizers also on smooth domain. Indeed, it was proved in [48, Example 3.4] that the $C^{1,1}$ function $u(x, y):=-2 x y+y|y|$ minimizes $\mathscr{A}$ (under boundary conditions given by $u$ itself) on any bounded domain $\Omega \subset \mathbb{R}^{2}$ with Lipschitz regular boundary. Notice that, by Remark 6.2 (b), $u$ satisfies a B.S.C. on any smooth and uniformly convex domain.

\section{References}

[1] L. Ambrosio, N. Fusco and D. Pallara, "Functions of Bounded Variation and Free Discontinuity Problems", Oxford Mathematical Monographs, The Clarendon Press, Oxford University Press, New York, 2000.

${ }^{2}$ Up to an easy adaptation. 
[2] P. BousQUeT, Boundary continuity of solutions to a basic problem in the calculus of variations, Adv. Calc. Var. 3 (2010), 1-27.

[3] P. Bousquet, Continuity of solutions of a problem in the calculus of variations, Calc. Var. Partial Differential Equations 41 (2011), 413-433.

[4] P. BousqueT and F. ClARKE, Local Lipschitz continuity of solutions to a problem in the calculus of variations, J. Differential Equations 243 (2007), 489-503.

[5] P. Bousquet, C. MARICONDA and G. TREU, Hölder continuity of solutions to a basic problem in the calculus of variations, C. R. Math. Acad. Sci. Paris 346 (2008), 1301-1305.

[6] L. CApogna, G. CitTi and M. ManfRedini, Regularity of non-characteristic minimal graphs in the Heisenberg group $\mathbb{H}^{1}$, Indiana Univ. Math. J. 58 (2009), 2115-2160.

[7] L. Capogna, G. CitTi and M. Manfredini, Smoothness of Lipschitz minimal intrinsic graphs in Heisenberg groups $\mathbb{H}^{n}, n>1$, J. Reine Angew. Math. 648 (2010), 75-110.

[8] A. CELlina, On the bounded slope condition and the validity of the Euler Lagrange equation, SIAM J. Control Optim. 40 (2001/02), 1270-1279 (electronic).

[9] A. CEllina, Comparison results and estimates on the gradient without strict convexity, SIAM J. Control Optim. 46, (2007), 738-749.

[10] A. CELlina, Uniqueness and comparison results for functionals depending on $\nabla u$ and on $u$, SIAM J. Optim. 18 (2007), 711-716 (electronic).

[11] A. Cellina, C. MARICONDA and G. TREU, Comparison results without strict convexity, Discrete Contin. Dyn. Syst. Ser. B 11, (2009), 57-65.

[12] J.-H. Cheng and J.-F. HwAng, Properly embedded and immersed minimal surfaces in the Heisenberg group, Bull. Aust. Math. Soc. 70 (2004), 507-520.

[13] J.-H. Cheng, J.-F. Hwang, A. Malchiodi and P. YAng, Minimal surfaces in pseudohermitian geometry and the Bernstein problem in the Heisenberg group, Ann. Sc. Norm. Super. Pisa Cl. Sci. 1 (2005), 129-177.

[14] J.-H. CHENG, J.-F. HWANG and P. YANG, Existence and uniqueness for p-area minimizers in the Heisenberg group, Math. Ann. 337 (2007), 253-293.

[15] J.-H. Cheng, J.-F. HwANG and P. YANG, Regularity of $C^{1}$ smooth surfaces with prescribed p-mean curvature in the Heisenberg group, Math. Ann. 344 (2009), 1-35.

[16] J.-H. CHENG and J.-F. HWANG, Variations of generalized area functionals and p-area minimizers of bounded variation in the Heisenberg group, Bull. Inst. Math. Acad. Sin. (N.S.) 5 (2010), 369-412.

[17] J.-H. Cheng, J.-F. HwAng, A. MAlchiodi and P. YANG, A Codazzi-like equation and the singular set for $C^{1}$ smooth surfaces in the Heisenberg group, J. Reine Angew. Math. 671 (2012), 131-198.

[18] J.-H. ChEng and J.-F. HWANG, Uniqueness of generalized p-area minimizers and integrability of a horizontal normal in the Heisenberg group, Calc. Var. Partial Differential Equations 50 (2014), 579-597.

[19] F. Clarke, Continuity of solutions to a basic problem in the calculus of variations, Ann. Sc. Norm. Super. Pisa Cl. Sci. (5) 4 (2005), 511-530.

[20] D. Danielli, N. Garofalo and D.-M. Nhieu, Sub-Riemannian calculus on hypersurfaces in Carnot groups, Adv. Math. 215 (2007), 292-378.

[21] D. Danielli, N. Garofalo and D.-M. Nhieu, A partial solution of the isoperimetric problem for the Heisenberg group, Forum Math. 20 (2008), 99-143.

[22] D. Danielli, N. Garofalo, D.-M. Nhieu and S. D. Pauls, The Bernstein problem for embedded surfaces in the Heisenberg group $\mathbb{H}^{1}$, Indiana Univ. Math. J. 59 (2010), 563-594.

[23] A. FIASCHI and G. TREU, The bounded slope condition for functionals depending on $x, u$, and $\nabla u$, SIAM J. Control Optim. 50 (2012), 991-1011.

[24] A. FiASCHI and G. TREU, Comparison principles, regularity and uniqueness results for minimizers in the $\mathrm{BV}$ space, in preparation.

[25] N. Garofalo and S. PAUls, The Bernstein problem in the Heisenberg group, preprint 2005, http://arxiv.org/PS_cache/math/pdf/0209/0209065v2.pdfarXiv:math/0209065v2. 
[26] E. Giusti, "Minimal Surfaces and Functions of Bounded Variation”, Birkhäuser, Boston, 1984.

[27] E. GiUsti, "Direct Methods in the Calculus of Variations", World Scientific Publishing Co., Inc., River Edge, NJ, 2003.

[28] A. HAAR, Über das Plateausche Problem, Math. Ann. 97 (1927), 124-158.

[29] P. Hartman, On the bounded slope condition, Pacific J. Math. 18 (1966), 495-511.

[30] P. Hartman, Convex sets and the bounded slope condition, Pacific J. Math. 25 (1968), 511-522.

[31] P. Hartman and L. Nirenberg, On spherical image maps whose Jacobians do not change sign, Amer. J. Math. 81 (1959) 901-920.

[32] G. J. HARTMAnN and G. Stampacchia, On some nonlinear elliptic differential equations, Acta Math. 115 (1966), 271-310.

[33] D. Hilbert, Über das Dirichletsche Prinzip, Math. Ann. 59 (1904), 161-186.

[34] R. K. HLADKy and S. D. Pauls, Constant mean curvature surfaces in sub-Riemannian spaces, J. Differential Geom. 79 (2008), 111-139.

[35] A. Hurtado, M. Ritoré and C. Rosales, The classification of complete stable areastationary surfaces in the Heisenberg group $\mathbb{H}^{1}$, Adv. Math. 224 (2010), 561-600.

[36] G. P. LEONARDI and S. RigOT, Isoperimetric sets on Carnot groups, Houston J. Math. 3 (2003), 609-637.

[37] C. MARICONDA, The lack of strict convexity and the validity of the comparison principle for a simple class of minimizers, Nonlinear Anal. 73 (2010), 828-834.

[38] C. MARICONDA and G. TREU, Existence and Lipschitz regularity for minima, Proc. Amer. Math. Soc. 130 (2002), 395-404 (electronic).

[39] C. MARICONDA and G. TREU, Lipschitz regularity for minima without strict convexity of the Lagrangian, J. Differential Equations 243 (2007), 388-413.

[40] C. MARICONDA and G. TREU, Local Lipschitz regularity of minima for a scalar problem of the calculus of variations, Commun. Contemp. Math. 10 (2008), 1129-1149.

[41] C. MARICONDA and G. TREU, Hölder regularity for a classical problem of the calculus of variations, Adv. Calc. Var. 2 (2009), 311-320.

[42] C. Mariconda and G. TReu, A Haar-Rado type theorem for minimizers in Sobolev spaces, ESAIM Control Optim. Calc. Var. 17 (2011), 1133-1143.

[43] M. MiRANDA, Un Teorema di esistenza e unicitá per il problema dell'area minima in $n$ variabili, Ann. Sc. Norm. Sup. Pisa (3) 19 (1965), 233-249.

[44] R. MonTi, Heisenberg isoperimetric problems. The axial case, Adv. Calc. Var. 1 (2008), 93-121.

[45] R. Monti and M. Rickly, Convex isoperimetric sets in the Heisenberg group, Ann. Scuola Norm. Sup. Pisa Cl. Sci. (5) 8 (2009), 391-415.

[46] S. D. PAuls, Minimal surfaces in the Heisenberg groups, Geom. Dedicata 104 (2004), 201-231.

[47] S. D. PAUls, H-minimal graphs of low regularity in $\mathbb{H}^{1}$, Comm. Math. Helv. 81 (2006), 337-381.

[48] M. RitORÉ, Examples of area-minimizing surfaces in the sub-Riemannian Heisenberg group $\mathbb{H}^{1}$ with low regularity, Calc. Var. Partial Differential Equations 34 (2009), 179-192.

[49] M. RitorÉ, A proof by calibration of an isoperimetric inequality in the Heisenberg group $\mathbb{H}^{1}$, Calc. Var. Partial Differential Equations 44 (2012), 47-60.

[50] M. Ritoré and C. Rosales, Rotationally invariant hypersurfaces with constant mean curvature in the Heisenberg group $\mathbb{H}^{n}$, J. Geom. Anal. 16 (2006), 703-720.

[51] M. RitorÉ and C. Rosales, Area-stationary surfaces in the Heisenberg group $\mathbb{H}^{1}$, Adv. Math. 219 (2008), 633-671.

[52] F. SERra CASSANO and D. VitTone, Graphs of bounded variation, existence and local boundedness of non-parametric minimal surfaces in Heisenberg groups, Adv. Calc. Var. 7 (2014), 409-492. 
[53] N. SHCHERBAKOVA, Minimal surfaces in sub-Riemannian manifolds and structure of their singular sets in (2, 3)-case, ESAIM Control Optim. Calc. Var. 15 (2009), 839-862.

[54] G. STAMPACCHIA, On some regular multiple integral problems in the calculus of variations, Comm. Pure Appl. Math. 16 (1963), 383-421.

Dipartimento di Matematica Università di Padova Via Trieste 63

35121 Padova, Italia pinamont@math.unipd.it treu@math.unipd.it vittone@math.unipd.it

Dipartimento di Matematica Università di Trento Via Sommarive 14 38123 Povo (TN), Italia cassano@science.unitn.it 